\title{
Analysis of Differences Between Human Alternative Splicing Protein Isoforms and Their Links to Diseases
}

by

\section{Haitham Hashim Mohammad Fallatah}

\author{
A thesis submitted to the \\ Faculty of Graduate and Postdoctoral Affairs \\ in partial fulfillment of the requirements for the degree of
}

\section{Master of Computer Science with Data Science Specialization}

Ottawa-Carleton Institute for Computer Science

Department of Computer Science

Carleton University

Ottawa, Ontario

September, 2019

(C) Copyright

Haitham Hashim Mohammed Fallatah, 2019 


\title{
Abstract
}

\begin{abstract}
Alternative Splicing (AS) is a process that is believed to have links to cellular function changes and some diseases in humans. Although AS was first discovered in the 1970s, not much research has been conducted on its role in functional implications on the proteome level. This study aims to use PIPE, a protein-protein interaction prediction algorithm, along with a tissue expression dataset to build a pipeline that differentiates between AS isoform products by analyzing isoform sequence changes, functional changes, and tissue expression changes that AS introduces. The study found that isoform sequence changes in alternative isoforms tend to be conserved deletions of amino-acid sub-sequences. The study also found that there is a statistically significant overlap between PIPE-predicted protein-protein interaction (PPI) network changes and tissue expression changes of alternatively spliced isoforms (ASIs) relative to their canonical isoforms (CIs) with a p-value of $8.25 \times 10^{-5}$. Finally, among the analysis pipeline top ten genes with predicted significant ASIs' PPI network changes, LMO2, THOC2, and UBE2L3 are genes that were suspected of having links to different diseases such as basel-type breast cancer, intellectual disability (ID) and numerous autoimmune diseases according to literature studies.
\end{abstract}




\section{Acknowledgments}

Firstly, I would like to thank my supervisor Dr. Frank Dehne for his outstanding support throughout my Master's degree. Dr. Dehne provided me with invaluable advice, support, and kindness during my Master's journey. I could not have asked for a better supervisor.

I would also like to extend my thanks to my co-supervisor, Dr. Ashkan Golshani whose support and advice have guided the scope of this thesis and helped me navigate through biochemical/biological concepts that I was not familiar with.

Secondly, I wanted to give a big thank you to the bioinformatics group members who listened, and provided advice on my presentations, specially Dr. Jim Green who provided me with feedback and support that was critical to the completion of this thesis.

A special thanks to Dr. Ashkan's students including Taylor for taking the time to meet with me on regular basis to discuss updates and provide research relevant material much of which was included in the thesis, and Narges for helping me with researching the literature to corroborate my results.

I also appreciate the support and patience that my family including my father Hashim 
Fallatah, my mom Zinab Snnary, and my four lovely sisters have provided me with before and after embarking on my thesis journey as I would have not been able to become who I am without them.

I am infinitely grateful to my wife and best friend Asha Siad, my wonderful sister Hiba Fallatah, and my dear friend Abdulrahman Al-Amoudi for taking the time to proofread this thesis.

I would like to also extend my appreciation for the Saudi Cultural Bureau on behalf of my country Saudi Arabia, for fully sponsoring my undergraduate studies which allowed me to pursue my Masters.

A final and sincere appreciation to Sylvain Pitre who is sadly no longer with us, but whose work on PIPE provides the cornerstone of my work and whose smile and pleasant company I miss. 


\section{List of Figures}

1.1 An example of how multiple protein isoforms with different combinations of exons and introns can be produced by alternative splicing [18]

1.2 Overview of the five basic splicing mechanism types. Exons are represented by yellow and blue blocks while introns are represented by the solid lines between exons $[33] \ldots \ldots \ldots \ldots$

1.3 This diagram shows the percentages of alternative splicing types that take place in some organisms. The organisms are divided in three different categories: invertebrates, vertebrates, and mammals. [43] . .

2.1 A flowchart detailing the steps taken in the network selection and combination step $[26] \ldots \ldots \ldots \ldots \ldots \ldots$

2.2 Overview of two methods major features depicted here as method A and method B $[26] \ldots \ldots \ldots \ldots \ldots$

2.3 Illustration of key isoforms of positive genes for a given function. At step (a), green dots are positive isoforms and blue dots are negative isoforms contained within genes (surrounding circles). At step (b) the algorithm tries to detect all target isoforms (green). At step (c) the algorithm draws the hyper-plane that separates positive and negative isoforms. $[31] \ldots \ldots \ldots \ldots \ldots \ldots$ 
2.4 Performance comparison of WLRM, miVLAD, miFV and miSVM [31]

2.5 Top: Machine Learning is used to infer a computational model of splicing, by correlating DNA elements with splicing levels in healthy human tissues. Bottom: genetic variants arising from a wide array of diseases and technologies can be detected and filtered using the computational model, enabling explorations into the genetics of disease [54] . . . . 25

2.6 Pipeline for the DIIP algorithm [12] . . . . . . . . . . . . . . . 27

2.7 An illustration of different types of isoform interaction profiles for a given pair of proteins $[12]$. . . . . . . . . . . . . . . . . 28

2.8 Process for comparative protein-protein interaction profiling [56] . . . 31

2.9 General overview of ISOexpresso workflow $[55] \ldots . . .33$

2.10 Three examples of expression levels for isoforms of genes of interest [55] 35

2.11 Steps taken by PIPE when comparing the sequences of two proteins $A$ and $B[40] \ldots \ldots \ldots \ldots$. . . . . . . . . . . . . . . . . . . . . . . . . .

3.1 Analysis pipeline overview . . . . . . . . . . . . . . . 42

3.2 Highest five human subcellular locations in terms of number of annotated AS genes . . . . . . . . . . . . . . . .

3.3 Frequencies of AS genes based on the number of alternatively spliced isoforms they have . . . . . . . . . . . . . . . . 46

3.4 Comparison between the full human set and the nucleus and chromosome subset in terms of frequencies of non-AS genes, AS genes, and

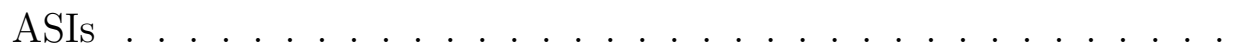

3.5 Comparison between the full human set and the nucleus and chromosome subset in terms of the types of sequence changes ASIs have relative to their CIs . . . . . . . . . . . . . . . . . . . 48 
3.6 Comparison between the full human set and the nucleus and chromosome subset in terms of the sequence alignment similarity scores of ASIs relative to their CIs . . . . . . . . . . . . . . . . . . . . . . . . . 49

3.7 Illustrative example of constructed PPI networks . . . . . . . . 53

3.8 Breakdown of significant ASI PPI networks based on the gain or loss of interaction partners when compared to their CIs PPI networks . . 56

3.9 Protein-Protein Network construction pipeline step overview . . . . . 58

3.10 Gene Ontology Terms divided by category types . . . . . . . . . . 60

3.11 GO annotations pipeline step overview . . . . . . . . . . . . 63

3.12 Frequencies of ASIs with significant over-expression/under-expression value changes for each of the 31 tissue types . . . . . . . . . . 66

3.13 Overview of tissue expression analysis pipeline step . . . . . . . . 67

3.14 Frequencies of overlapped over-expressed/under-expressed ASIs for each of the 31 tissue types (90\%, 95\%, 99\% confidence levels are represented by $^{\prime}{ }^{\prime},{ }^{\prime * *}$, , and ${ }^{\prime * * *}{ }^{\prime}$ respectively) . . . . . . . . . . . . 


\section{List of Tables}

$3.1 A S I_{A}$ and $C I_{x}$ PPI networks comparison $\ldots \ldots \ldots \ldots$

$3.2 A S I_{B}$ and $C I_{x}$ PPI networks comparison $\ldots \ldots \ldots \ldots$

3.3 Top 10 ASIs based on AS PPI network scores . . . . . . . . . 57 


\title{
List of Abbreviations
}

\author{
CI Canonical isoform \\ ASI Alternatively spliced isoform \\ AS Alternative splicing \\ AS gene A gene that undergoes alternative splicing and produces at least one \\ ASI \\ AS CI A canonical isoform of an AS gene. \\ non-AS gene A gene that does not undergo alternative splicing. \\ non-AS CI A canonical isoform of a non-AS gene \\ PPI Protein-Protein interaction \\ IP Interaction partner/protein
}




\section{Contents}

$\begin{array}{ll}\text { Abstract } & \text { i }\end{array}$

Acknowledgements $\quad$ ii

List of Figures $\quad$ vi

List of Tables vii

List of Abbreviations viii

1 Introduction 1

1.1 Background and Motivation . . . . . . . . . . . . . . 1

1.2 Thesis Objective . . . . . . . . . . . . . . . . . . . . 10

1.3 Thesis Organization . . . . . . . . . . . . . . . . . 10

2 Literature Review $\quad 12$

2.1 Computational Approaches . . . . . . . . . . . . . . . . . . . . . . 12

2.1.1 MIL-Based Methods ... . . . . . . . . . . . . . . 14

2.1.2 Other Computational Methods ............. . 23

2.2 RNA-seq Non-Computational Analysis Approaches . . . . . . . . . 29

2.2.1 Yeast-two-Hybrid Study . . . . . . . . . . . . . . . . 29 
2.2.2 ISOexpresso, alternative splicing, and cancer . . . . . . . . 32

2.3 PIPE Algorithm Review . . . . . . . . . . . . . . . . . 36

3 Alternative Splicing Effects Analysis $\quad 39$

3.1 Pipeline Overview . . . . . . . . . . . . . . . . . . . . . . . . . . . . 39

3.2 Datasets collection \& cleanup . . . . . . . . . . . . . . . . . 43

3.3 Dataset Analysis . . . . . . . . . . . . . . . . . . . . 44

3.4 Protein-Protein Interaction Networks Construction _ . . . . . . . 50

3.4 .1 Introduction . . . . . . . . . . . . . . . 50

3.4.2 PIPE Setup and Predictions of PPIs . . . . . . . . 50

3.4.3 Comparing Alternatively Spliced Genes' Isoforms . . . . . . . 51

3.4 .4 Illustrative Example . . . . . . . . . . . . . . . . . . 53

3.4.5 Results \& Discussion . . . . . . . . . . . . . . . . . 55

3.5 Gene Ontology Term-Finder . . . . . . . . . . . . . . . . . . 59

3.5 .1 Introduction . . . . . . . . . . . . . . . . . 59

3.5.2 Dataset . . . . . . . . . . . . . . . . . . 59

3.5.3 GO Term Finder . . . . . . . . . . . . . . . . . . . . 59

3.6 Tissue Expression Analysis . . . . . . . . . . . . . . . . . . . . . 62

3.6 .1 Introduction . . . . . . . . . . . . . . . . 62

3.6 .2 Dataset . . . . . . . . . . . . . . . 64

3.6.3 Dataset-cleanup \& Analysis steps . . . . . . . . . . . . . . 64

3.6.4 Results \& Discussion . . . . . . . . . . . . . . . 65

3.7 PPI networks \& Tissue Expression Results Overlap . . . . . . . . . 67

3.7 .1 Introduction . . . . . . . . . . . . . . . . . 67

3.7 .2 Example . . . . . . . . . . . . . . . . . . . . . . 68

3.7 .3 Overlap Types . . . . . . . . . . . . . . . 68

3.7.4 Statistical Significance Calculation Method . . . . . . . . . 70 
3.7.5 Results \& Discussion . . . . . . . . . . . . . . . . . . 71

3.8 Pipeline \& Literature Results Overlap . . . . . . . . . . . . . . . . . . 74

3.8.1 Introduction . . . . . . . . . . . . . . . . . . . 74

3.8.2 Top Ten Literature Overlapped ASIs . . . . . . . . . . . . . . 74

4 Challenges, Conclusion, and Future Work $\quad 79$

4.1 Challenges . . . . . . . . . . . . . . . . . . . 79

4.2 Conclusion . . . . . . . . . . . . . . . . . . . 80

4.3 Future Work . . . . . . . . . . . . . . . . . . . . . 82 


\section{Chapter 1}

\section{Introduction}

\subsection{Background and Motivation}

Living organisms' biochemical systems are among the most complicated and fascinating systems to study and understand. All functions and processes that occur within a living organism are due to some form of a biochemical reaction. Examples include conversion of food to energy, cell building and repair, fighting foreign objects, among other functions. Most of these functions are performed by biochemical molecules called proteins. Proteins are constructed by a chain of building blocks called amino acids that form sequences that ultimately give proteins different structures, and as a consequence, different cellular functions [42]. Proteins' functional diversity is influenced by the genes that encode them.

Genes are contiguous parts of DNA sequences that contain the unique genetic code for each living organism. Genes are the means by which the cell machinery is able to encode different types of proteins and give them a variety of structures and func- 
tions. The human genome for example, has about 20,000 coding genes. According to the one-gene one-polypeptide hypothesis proposed by Edward Tatum in 1941, each gene corresponded to one polypeptide (i.e. protein), but later studies showed that through a process called alternative splicing, a gene can produce more than one protein $[17][56][23]$.

Alternative splicing (or simply AS) is a post-transcription process that allows multiple proteins, or protein isoforms, to arise from a single gene. AS is closely linked to the central dogma in molecular biology and thus it is imperative to shed some light on this process.

The central dogma explains the process of how the genetic information that is contained within an organisms' DNA can be used to synthesize proteins through two subsequent steps[10][45]. First, a region of DNA that corresponds to a protein-coding gene is converted into a messenger RNA (mRNA) molecule. This step is called transcription and takes place in the nucleolus. Following the completion of transcription, a subsequent step called translation takes place in the cytoplasm. Through translation, an mRNA strand is decoded and is used as a template for creating an amino acid sequence that undergoes additional post-translation steps eventually rendering it into a fully functional protein [10][45].

mRNA is the molecule through which AS can produce alternative isoforms of a given gene. An mRNA strand is composed of exons and introns. Exons are the parts of an mRNA sequence that contain protein-coding regions that can possibly be included in the mature mRNA sequence while introns are sequence regions that are spliced-out and are not included in the final mRNA sequence [10][45]. AS produces alternative 
isoforms through the process of modifying the mRNA sequence by excluding or including different combinations of exons in the final mRNA molecule [56] [23]. Figure 1.1 outlines the basic idea of AS.

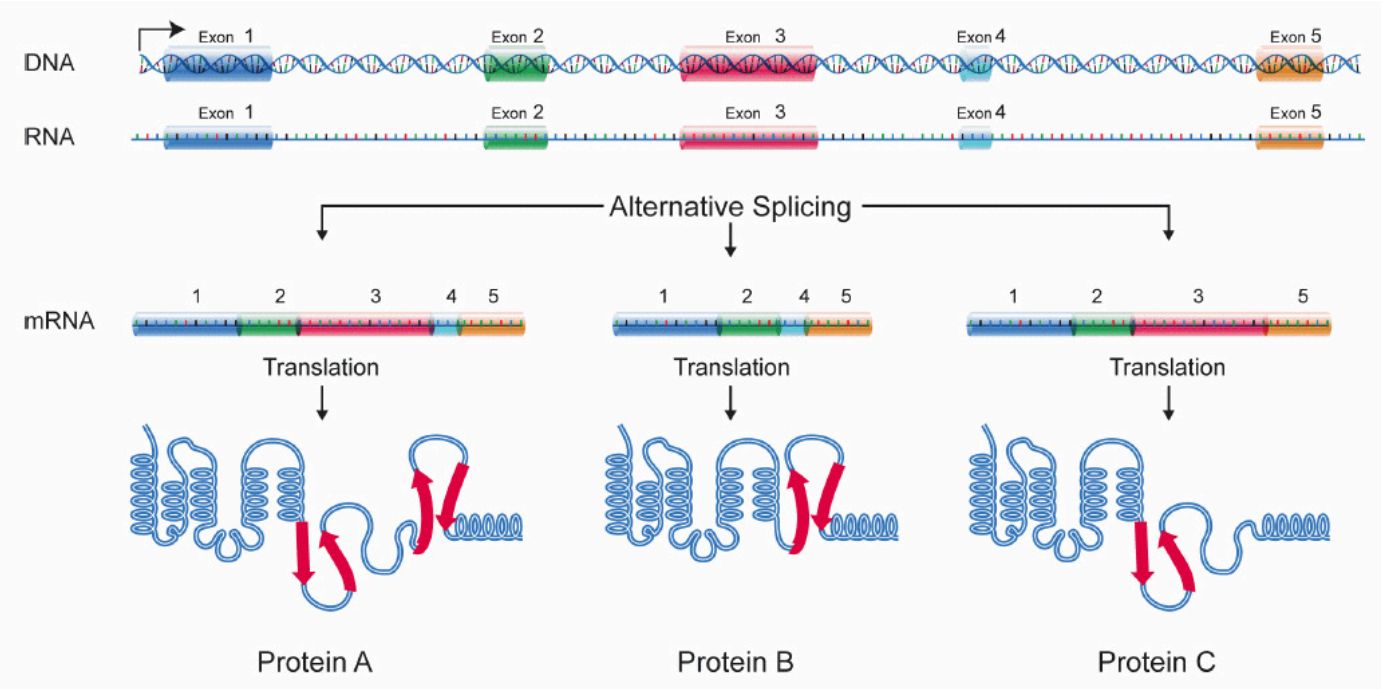

Figure 1.1: An example of how multiple protein isoforms with different combinations of exons and introns can be produced by alternative splicing [18]

The process of alternative splicing was first discovered in the 1970s when researchers from MIT (Massachusetts Institute of Technology) observed that a virus called Adenovirus produces six different transcripts after infiltrating and infecting a cell [9][3]. In a study published in 1986, a group of researchers found that the AS process (the process was called "Alternative RNA Processing" in the paper) can also occur in Eukaryote mammalian genes. The researchers observed that two genes that encode a thyroid hormone, namely PPT and CGRP, were producing multiple mRNA transcripts $[20]$.

There are five basic splicing mechanisms by which alternative splicing produces differ- 
ent versions of mature mRNA transcripts. The five mechanisms include exon skipping, mutually exclusive exons, alternative donor sites, alternative acceptor sites, and intron retention [43]. Exon skipping occurs when a given exon is excluded from the final mRNA transcript. The mutually exclusive exons splicing type occurs when one of two given exons is included in the final mRNA transcript but not the other exon. Alternative $5^{\prime}$ donor site occurs when the original 5' splice site of an exon is replaced by an alternative $5^{\prime}$ splice site as a result of a mutation thus changing the length of the exon. For the alternative 3 ' acceptor site splicing type, the same procedure as the alternative $5^{\prime}$ donor site type occurs but with the alternative $3^{\prime}$ splicing site instead. Finally, intron retention occurs when an intron is retained in the mature mRNA product [43]. Figure 1.2 illustrates the basic five AS splicing mechanisms. 


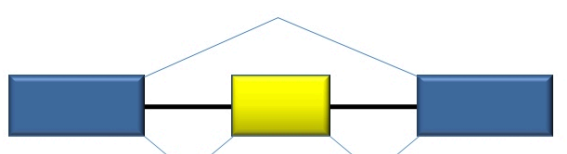

Exon skipping

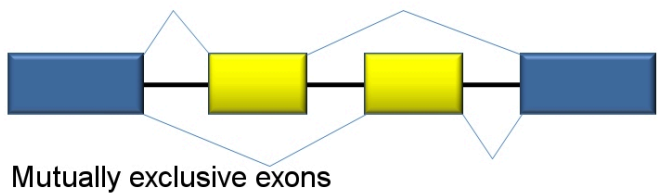

Mutually exclusive exons

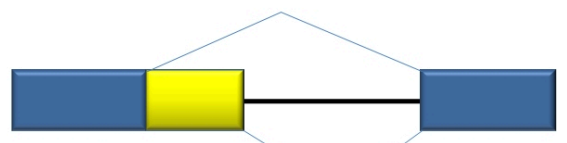

Alternative $5^{\prime}$ donor sites

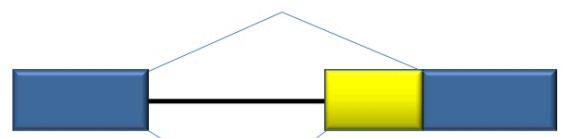

Alternative $3^{\prime}$ acceptor sites

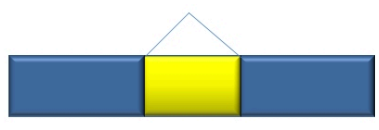

Intron retention

Figure 1.2: Overview of the five basic splicing mechanism types. Exons are represented by yellow and blue blocks while introns are represented by the solid lines between exons [33]

The five AS splicing mechanism types take place with varying proportions within different organisms. In mammals for example, exon skipping is the most common type of splicing while uncategorized/non-structured splicing tends to be the most common in invertebrates [43]. Refer to Figure 1.3 for more details. 


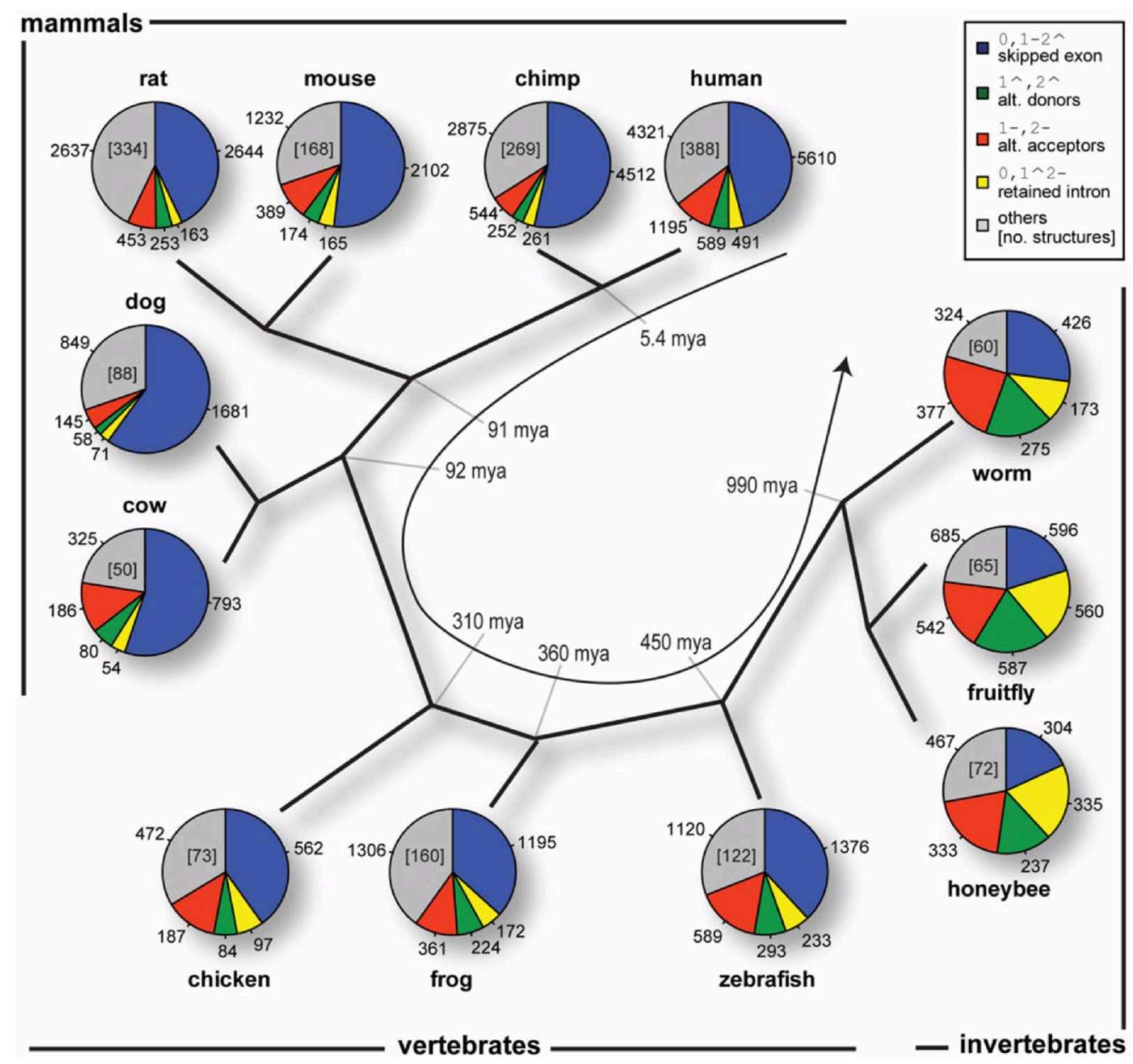

Figure 1.3: This diagram shows the percentages of alternative splicing types that take place in some organisms. The organisms are divided in three different categories: invertebrates, vertebrates, and mammals. [43]

After alternative splicing produces the final transcripts of a given gene, the long standing challenge of identifying which transcript is the canonical isoform (CI) and which transcripts are the alternatively spliced isoforms (non-canonical or ASI) surfaces [21]. Several definitions exist for describing what a canonical isoform is. For example, the isoform with the clearest domain definitions, the most conserved isoform among multiple species, the most expressed isoform, or simply the longest isoform are some of 
the factors used by various databases to label an isoform as canonical [1][41][14][21]. Ultimately, there is no consensus among researchers as to what defines a canonical isoform and thus, the characterization of a CI and an ASI of a gene remains somewhat arbitrary and database-dependent [21].

The occurrence of alternative splicing is quite prevalent in the cell. It is believed that more than $90 \%$ - 95\% of human genes undergo AS and produce multiple transcripts [22][26][21]. This can help explain the fact that the human genome and the genome of much smaller and simpler organisms such as fruit flies are comparable in number yet vary greatly in functional complexity [56]. Through alternative splicing, the number of proteins that can be produced from the human genome (which has 20,000 genes) can reach up to 100,000 , with as many as 82 proteins per gene [22].

Many broader aspects of AS remain unknown and need more research. For example, $\mathrm{AS}$ is known to diversify the functional complexity of genes; however, the extent of this diversification on the full proteomic level remains largely unknown [56]. In addition, some research questions such as the degree of functional diversification that AS exerts based on the organism type, or the different roles AS may play in the process of evolution are examples of questions that need further investigation [56]. Also, when it comes to investigating functions at the isoform level, no golden function-labeling standard for isoforms exists which makes it difficult for researchers to annotate functions to isoforms $[31][36]$.

The introduction and wide use of sequencing technologies such as RNA-seq, have been key in accelerating the research of alternative splicing and its implications [22]. Similar to micro-array data, RNA-seq covers different types of tissues and cell linings; however, RNA-seq is capable of detecting expression data at the isoform level more efficiently than micro-arrays [22]. Deep sequencing of RNA transcripts in tissue 
samples provides a wealth of data to study AS and to be able to differentiate isoforms based on expression levels [31]. Many methods have been developed to precisely measure the expression levels for each transcript; thus, allowing for the transfer of functional annotations from the gene level to the isoform level.[26].

Studying alternative splicing allows us to better understand the many implications that AS has over biological processes that are linked to functional diversity of isoforms and diseases. As for the functional diversity of isoforms, some studies showed that some genes are capable of executing multiple functions in the cell [22]. This phenomenon is related to whether the gene with multiple functions has different AS products or not. In some cases, isoforms arising from the same gene may exhibit contrasting functions to an extent where the isoforms could be considered to have come from different genes [22][21]. For example, in the case of the 2-isoform gene Bcl-x, one of its isoform has an anti-apoptotic function while the other isoform of the same gene is pro-apoptotic [22]. Researchers also found that there is a correlation between the number of protein interactions a given gene is reported to be involved in and the number of alternatively spliced products it can produce [12]. This is further evidence that AS can explain the functional complexities of organisms. It also indicates that AS is a tightly regulated process whose products may vary depending on different cell conditions [37].

Alternative splicing has also been found to be linked to different cases of diseases such as cancer and autism [22][54]. Aberrant splicing conditions may cause dysfunctions in the process of $\mathrm{AS}$ which results in products that have structural defects or abnormal expression levels ultimately leading to disease. Many studies and examples of cancer-causing splicing events exist [22][55][21][54]. For example, a study showed that BRCA1, a gene associated with breast cancer, is susceptible to an AS event that 
leads to the removal of the 11th exon and thus producing an isoform that causes breast cancer as a result of an imbalance in isoform products [47]. Another example is gene P53 which is a well-known tumor suppressor. P53 produces multiple variants including two that are associated with cancer cases, namely p53 (beta) and (delta) 133p53. The former isoform promotes apoptosis while the latter inhibits it. Irregular splicing patterns causes an irregular expression levels of both isoforms causing cancer proliferation [5]. Other examples of genes with links to cancer include CD99, CASP3, and NEK2 [44][52][30].

Besides cancer, alternative splicing has also been found to be linked to age-related diseases [24]. Aging is a main factor in many chronic diseases such as diabetes, hypertension, and neurodegenerative diseases [24]. Mutations occurring on or around splicing sites cause abnormal AS patterns that produce defective disease-causing isoform products. There are three main mechanisms by which AS can cause some of the age-related diseases. The first is when AS produces an isoform with impaired or degraded function that replaces the wild-type isoform. EAAT2 is an example gene that causes ALS as a result of this erroneous first mechanism [27]. The second mechanism is when AS gives rise to isoforms that are truncated versions of the normal isoform that are harmful to cell functions. A truncated isoform variant that is produced from a gene called LMNA is responsible for pre-mature aging in humans [11]. Finally, the third mechanism occurs when ratios of same-gene-isoforms that have collaborative functions that are produced from the same gene are abnormal as a result of a defective AS event. TAU is a gene that has two isoforms 3R-tue and 4R-tue. Having irregular ratios of these isoforms causes dementia [13]. 


\section{$1.2 \quad$ Thesis Objective}

This thesis is an exploratory project that aims to provide a better understanding of alternative splicing to researchers who study AS isoform products and may be interested in the functional or expression profile differences between alternatively spliced isoforms and their canonical isoforms. Another aim of the study is to investigate the possible presence of links between AS products'functional and tissue expression changes, and AS-linked diseases.

The scope of this study will focus on four aspects of alternative splicing. First, we start with a general analysis of differences and similarities among AS human genes and sequences. The second aspect covers analyzing some AS effects on ASI PPI networks relative to their CIs PPI networks. The third aspect focuses on tissue expression changes between ASIs and their CIs. The last aspect covered in this study focuses on reviewing the study results in light of experimentally-validated results from the literature.

\subsection{Thesis Organization}

This thesis is divided into four main chapters. In the first chapter we dive into the biology background of alternative splicing including its discovery, mechanisms, and its implications for diseases and functional diversity in organisms. In the second chapter, we review alternative splicing analysis techniques that are currently used in the field. The review covers computational methods, RNA-seq based analysis methods, and other studies related to alternative splicing and its disease implications. At the end of the chapter, the PIPE algorithm that is used in this project is reviewed. The 
third chapter presents the analysis pipeline that was used to obtain the results of this project and the significance of its results. The areas that were covered by the analysis pipeline include alternative splicing and its implications on protein-protein interaction networks, AS and its implications on tissue expression, and the overlap of cases from this study's results with cases from the literature. Finally, the last chapter summarizes the results and outlines some of the future directions that this project can be expanded into. 


\section{Chapter 2}

\section{Literature Review}

\subsection{Computational Approaches}

Recent advancements in the speed of computational processing and storage resources have moved research forward in the field of bioinformatics. Lab validations of experimental results are often expensive, time-consuming, and in some cases impractical [22]. Performing in-silico experiments or computational simulations on the other hand, is relatively cheap, fast, and scalable.

In the case of alternative splicing isoform products functional annotations, computational methods have been integral in advancing our understanding of them [31][36][12][54]. Before the large-scale generation of RNA-seq data, protein isoforms functional annotations were initially produced based on protein-structure prediction tools such as I-TASSER [22]. 
The iterative threading assembly refinement server (I-TASSER) is a tool that automates the process of protein structure determination and subsequently predicts its function [42]. The algorithm uses protein primary sequences to generate a 3D model by running iterative structural simulations. The function of the protein is then inferred from known protein structures that resemble the predicted protein structure $[42]$.

In a study conducted by Menon et al. [22], I-TASSER was used to predict different isoform functions that arise from genes which are known to have differential isoform expression profiles in mice that have cancer. able to find biologically relevant motifs that are suspected to cause functional difference within five genes (Anxa6, Ptbp1, Tax1bp3, Calu, and Cdc42) [22].

Although in some cases, structural-based function prediction can successfully identify functional differences between isoforms, this method still has its limitations. One major limitation is that it relies on the protein structures that are known. This would prevent the algorithm from being able to match the structure of a protein that does not resemble any structure in the database [22]. Moreover, another limitation concerning the function prediction step is that in many cases, isoforms have subtle differences in motifs or domains which are enough to cause functional differences that are not detectable by structure prediction algorithms [22].

With the recent wide availability of RNA-seq data, many researchers have capitalized on the abundance of expression datasets and attempted to design machine learning models that are capable of identifying protein isoforms functions and mending the gap of structure prediction algorithms. In the following section, we review and examine some of these methods. 


\subsubsection{MIL-Based Methods}

Multiple-Instance Learning (MIL) is a supervised machine learning technique [2]. Rather than training on labeled individual instances, it deals with a group of instances that are collectively labeled based on the labeling of its instances. In its simplest form, the group of instances is called a bag, where a bag will either have a positive label if at least one positive instance within it exists, or will be labeled negative otherwise [2].

Consider the following example of a MIL problem. Imagine having three key chains (bags) each with a separate set of keys (instances). Assume that key sets one and three (positive bags) can open a particular lock while key set two cannot (negative bag). The problem to be solved here is the ability to predict which key (or keys) within key sets one and three are the keys that are common among the two sets and can open the lock [2].

There are many computational approaches that provide the mechanism that allows for the prediction of the instance or instances of interest that are defined by an MILtype problem. Some approaches employ mathematical, or statistical methods such as logistical regression, or maximum likelihood, or more machine learning based methods such as SVM [31][36][26][2].

This machine learning technique has the ability to solve problems in many fields where there is a hierarchy between the pieces of the problem to be predicted or when there is a parent child relationship. Furthermore, examples of applications of MIL can be found in the field of computer vision analysis when comparing similar pictures, in chemistry when distinguishing similar molecules, or in bioinformatics when assigning functional labels for alternatively spliced isoforms of a given gene [22]. 
In the following sections, a review is presented for some of the algorithms that use the MIL paradigm to solve the problem of labeling alternatively spliced isoforms of a given gene to a function.

\section{Instance-Oriented MI Label Propagation Model}

Li et al. introduced a machine learning algorithm that is based on the concept of multiple-instance learning called instance-oriented MI Label Propagation (iMILP) to predict the function of individual isoforms [25]. iMILP combines MIL concepts along graph-based Label Propagation (LP) methods [4]. More specifically, the algorithm propagates labels from annotated instances to unannotated instances through edges of a graph. Contrary to traditional LP algorithms, iMILP can have a collection of instances where the label of the collection is shared with at least one of its instances [25][2]. In our example of gene-isoform relationship, iMILP can have a gene label with function $\mathrm{X}$ when at least one of its isoforms is labeled with the same function $\mathrm{X}[26]$.

The algorithm works by either of two methods which predict isoform functions [25][26]. The first method contains two steps. The first step is the network selection and combination step. In this step, the algorithm takes multiple co-expression networks of protein isoforms and based on a group of functions of interest, the algorithm selects the best subset network for each function and aggregates it with the other subset networks of other functions. The algorithm iterates over all given functions until it forms one single co-expression network at the end [26].

The second step is the prediction step where the algorithm takes the co-expression network produced in the previous step and tries to assign functions to protein iso- 
forms. Each bag (gene) of isoforms is initialized with a value of $1,-1$, or 0 with the values representing having a given function, not having a given function, or being undetermined in regards to having or not having a given function respectively. Using an adjacency matrix representing isoform-isoform weight associations, the algorithm iteratively updates the values of each isoform based on the edges of the co-expression network and propagates function annotations across the network until the algorithm converges and each isoform in the network is assigned a function [26]. Figure 2.1 illustrates the general overview of the algorithm.

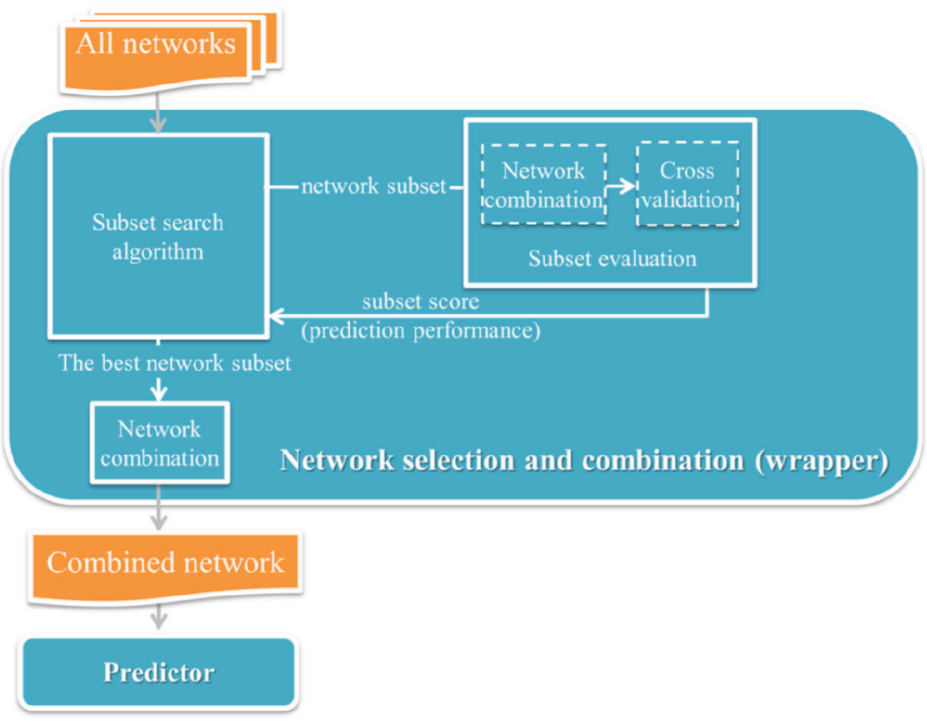

Figure 2.1: A flowchart detailing the steps taken in the network selection and combination step [26]

The second method merges co-expression networks in addition to Domain-Domain interaction datasets to construct a genome wide isoform-isoform interaction network. This network is then used to identify dense clusters where isoforms are heavily con- 
nected. Isoforms in these clusters would then be assigned functions according to function labels present in the cluster [26]. An outline of both methods is depicted on Figure 2.2.
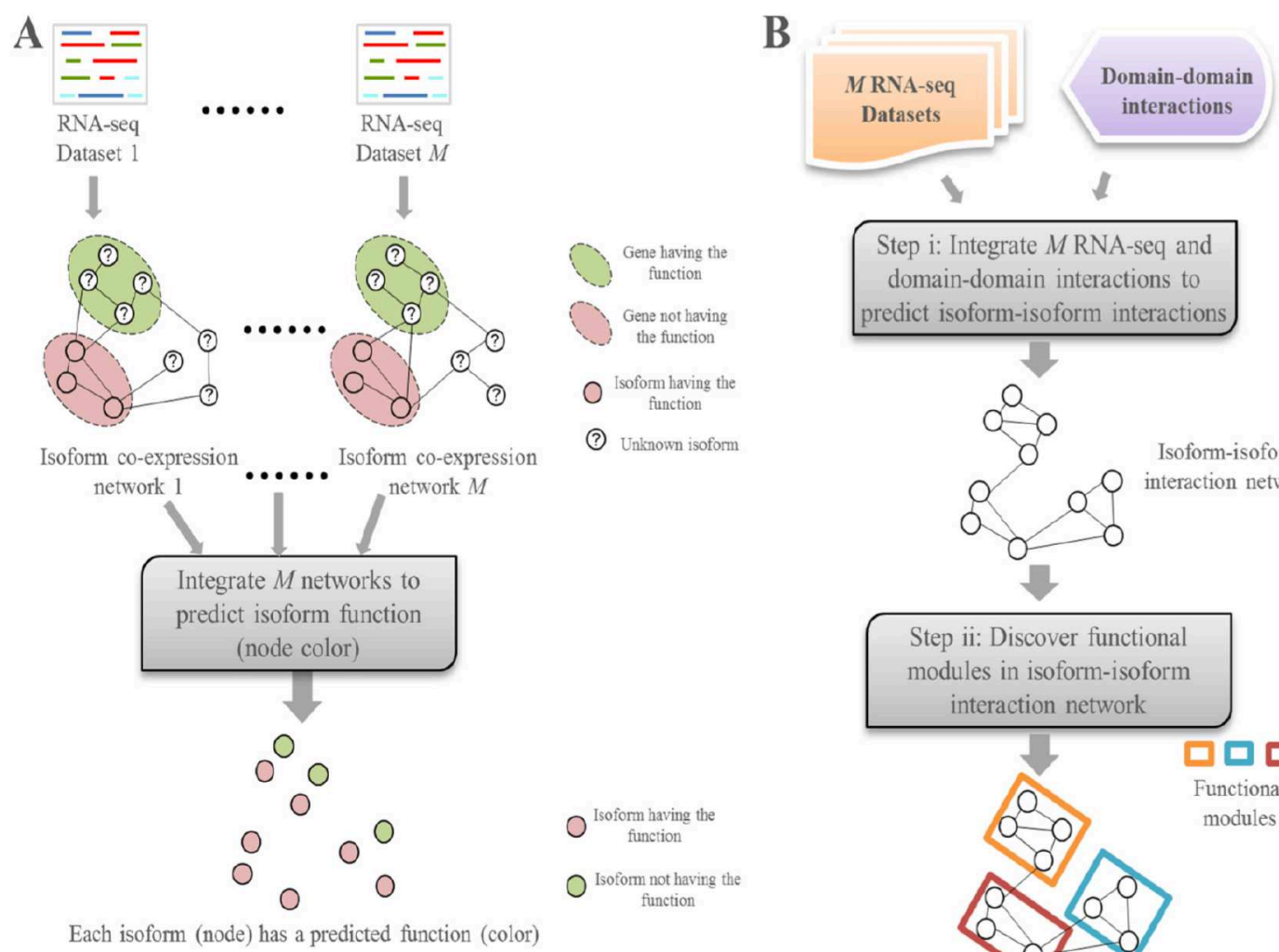

Step i: Integrate $M$ RNA-seq and domain-domain interactions to predict isoform-isoform interactions
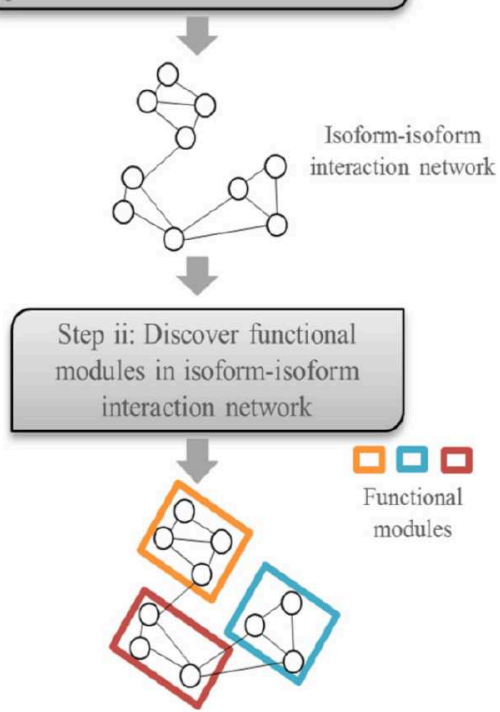

Figure 2.2: Overview of two methods major features depicted here as method $A$ and method $B$ [26]

The authors mentioned that the first method was able to annotate 70,392 functions to 15,572 isoforms. Function annotations for genes come from the GO ontology database. This method achieved a precision score of 0.67 when assessed by the Area Under the 
Curve (AUC) performance measure [26]. Also, the algorithm was able to correctly label 11 well-reviewed isoforms that belong to the genes TP53, BCL2L1, CFLAR, CASP8, and DNAJA3 with the function regulation of apoptotic process [26].

The algorithm puts more emphasis on trying to predict whether an isoform inherits the function of its gene rather than finding out if an isoform gains new functions [26]. Additionally, the algorithm from this paper uses 29 RNA-seq datasets to calculate co-expression networks which could be computationally expensive [22].

\section{Weighted Logistic Regression-Based Multi-Instance Learning}

In a paper authored by Luo et al. a novel approach called Weighted Logistic Regressionbased Multi-Instance Learning was introduced to assign functional annotations to isoforms of a given gene [31]. Many multi-isoform genes perform multiple functions that are often performed by a subset of their protein coding isoforms. Protein isoforms that inherit functions from their gene are called key isoforms. The authors in this paper try to identify which isoforms of genes are labeled with a given function, are key protein isoforms that are closely related to the given function and which ones are not. Figure 2.3 illustrates the main task of the algorithm. 


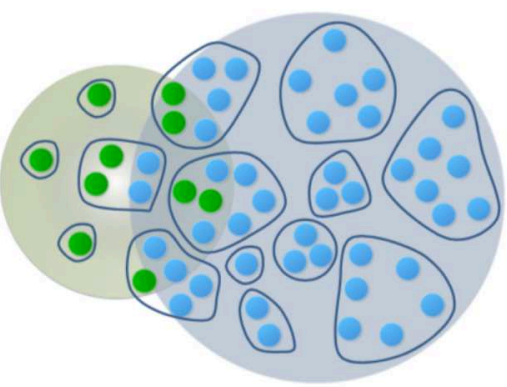

(a)

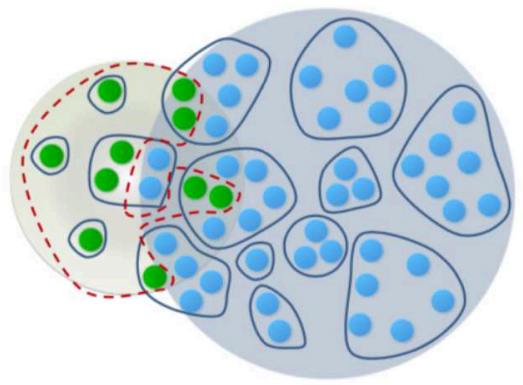

(b)

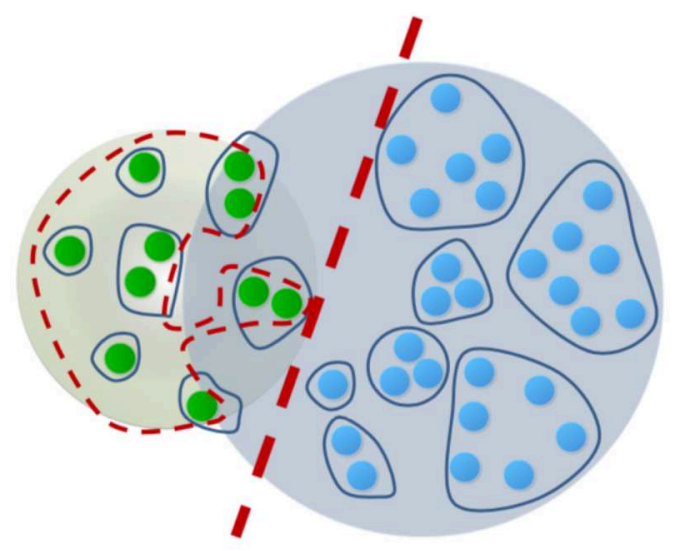

(c)

Figure 2.3: Illustration of key isoforms of positive genes for a given function. At step (a), green dots are positive isoforms and blue dots are negative isoforms contained within genes (surrounding circles). At step (b) the algorithm tries to detect all target isoforms (green). At step (c) the algorithm draws the hyper-plane that separates positive and negative isoforms. [31]

Given a group of genes along with their protein isoforms, and a function to be queried, the algorithm starts by defining genes as bags and protein isoforms as instances within 
each bag. Each gene bag is assigned as either a positive bag (for genes that are known to have the function of interest) or a negative bag (for a gene that does not have the function of interest) [31].

A statistical method called non-convex sparsity-inducing, is at the core of this computational model [31]. Sparsity regularization is a statistical approach that attempts to reduce the search space of a problem by the use of a loss function. For this context, it is used to assign a function label to a subset of key protein coding isoforms that exhibit this function across all positive genes. The model uses existing functional gene labels based on the GO functional term annotations [31].

With the assumption that each positive gene bag contains at least one protein isoform that has the function of interest, the algorithm then proceeds to employ the statistical model to assign weights to each isoform and continue to update the weights in a way that maximizes the difference between key isoforms and negative isoforms until the algorithm converges and key isoforms within positive genes are identified [31]. The algorithm achieves this by using protein isoforms RNA-seq data.

To validate the effectiveness of their model, the authors used an RNA-seq dataset for humans to extract the expression results of 59,287 protein isoforms from 11,946 genes [31]. The model can work with 94 benchmark GO-term gene annotations that were selected by the authors. These 94 terms were then further divided into five groups based on how many genes they are labeled with (A, B, C, D, and E ascendingly). 5fold cross validation was then used as a validation measure for the models prediction performance [31]. 
The results of the computational model are demonstrated in Figure 2.4 where the authors compared the performance of their model by standard machine learning performance measures such as finding the Area Under the ROC curve, specificity, and sensitivity to three other similar tools in the field [31].
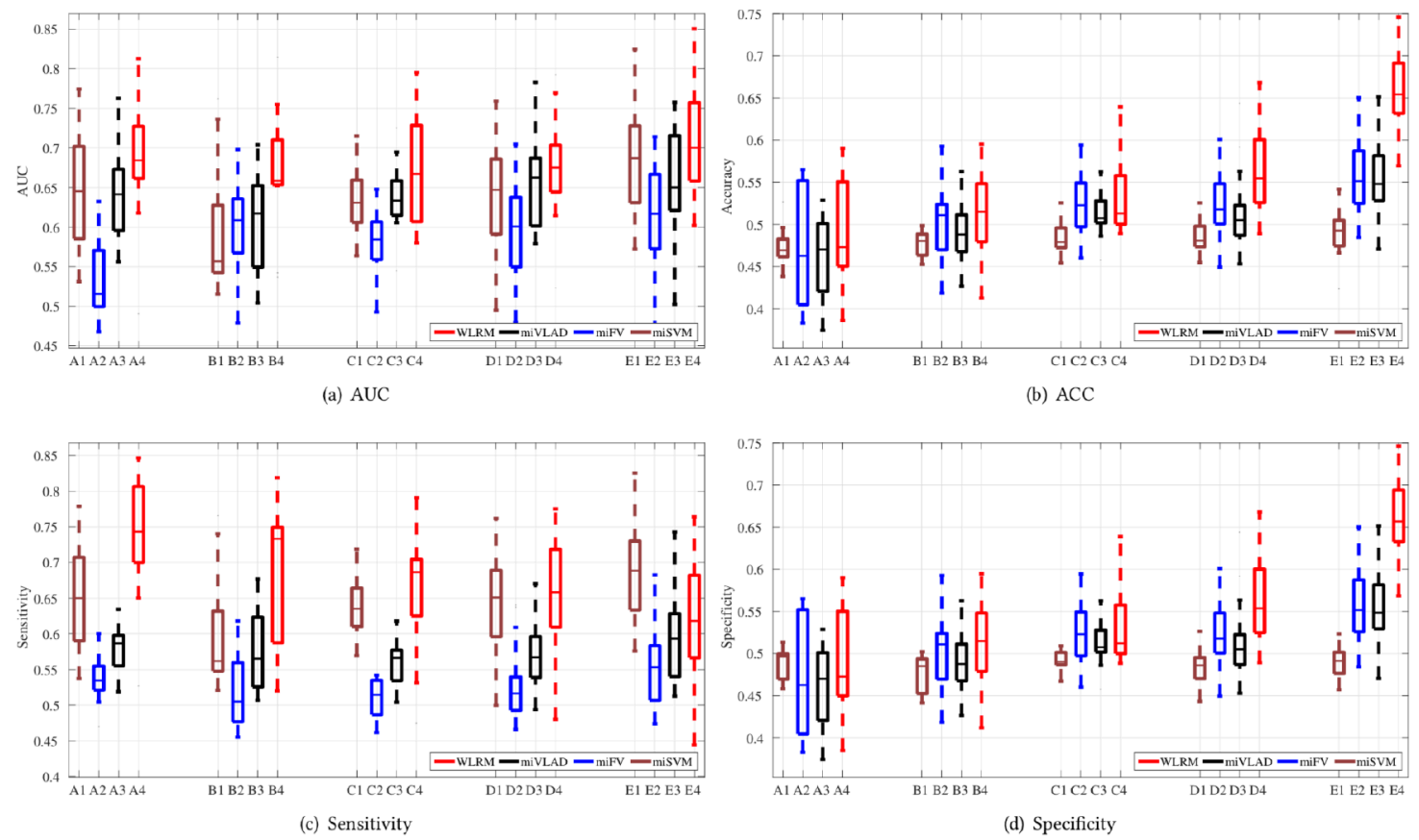

Figure 2.4: Performance comparison of WLRM, miVLAD, miFV and miSVM [31]

Although the algorithm is successful in identifying key isoforms in each gene, the scope of the paper is limited. The model does not cover the identification of functions of isoforms that are labeled negatively under a given gene; hence, it lacks the ability to be able to discover novel isoform function annotations. Another limitation is the limited number of supported function annotations that the model can handle. WLRM can only handle 94 function annotations which is a small fraction of the total number of GO Terms annotations [31]. 


\section{IsoFunc}

A study authored by Panwar et al. used an MIL-SVM based approach to maximize the functional differences between protein coding splice variants (PCSV) in positive gene bags and other PCSVs [36].

The algorithm proposed is initially presented with bags of genes and instances of isoforms within those genes. The goal of the algorithm is to be able to distinguish isoforms of a given gene in relation to a given function [36].

A selection of 2,129 GO terms was used in the study [36]. They were divided into five groups depending on how many genes they are labeled with. The groups including the range of annotated genes are A (20-27), B (28-40), C (41-64), D (65-114) and E (115-300) [36]. Based on the RNA-seq data used for this study, the authors were able to extract 11,946 genes and 59,297 PCSVs expression data to train and test their model [36].

A 5-fold cross-validation was used to measure the prediction performance of the model while the Area Under the Curve (AUC) was used as the standard performance measure in this study. The authors found that the more PCSVs a gene had, the higher the performance achieved when predicting the function of PCSVs of that gene [36].

The authors used two genes and their PCSVs that have experimental data at the isoform level for validating the effectiveness of their SVM MIL-based approach. ADAM15 is a gene known to be involved in cell adhesion. The study found that the protein isoforms of this gene (ADAM15A, and ADMA15B) affect cell adhesion in different ways. Cell adhesion was enhanced by ADAM15A whereas reduced by ADAM15B. The algorithm introduced by the authors corroborated these results [36]. 
LMNA/C is a gene that plays an important role in nuclear assembly and has three main protein isoforms (lamin A, progerin, and lamin C). According to the expression data, lamin $\mathrm{C}$ was found to be associated with prolonged life in mice [36]. The algorithm showed that major GO terms changes related to metabolism for lamin A, and progerin, but none for lamin C [36].

\subsubsection{Other Computational Methods}

Although MIL-Based computational algorithms are the widely used method for annotating functions at the isoform level, other computational methods have been used to try to associate functional labels to protein isoforms or linking alternative splicing products to diseases. In the following two sections, we review two of these methods, and their findings.

\section{Deep Learning Based Method}

Xiong et al. presented a deep learning approach that assesses DNA sequences for disease-linked DNA variants that affect mRNA splicing events [54]. Abnormal splicing events that occur in mRNA transcripts as a result of changes in genetic variants are linked to many diseases [54]. In their study, Xiong et al. were able to discover links between some genetic variants and the occurrence of diseases such as spinal muscular atrophy, hereditary colorectal cancer, and autism spectrum disorder.

The algorithm applies general rules of splicing to find splicing sites and subsequently using Bayesian networks to construct a model that is capable of predicting and assigning scores to variants based on their likelihood of affecting mRNA splicing events [54]. 
The training step of the prediction model is not dependent on data coming from known disease-linked DNA variants; hence, it is less susceptible to bias. RNA-seq data was also used by the algorithm to compare splicing levels in conjunction with the presence or absence of a given DNA variant. See Figure 2.5 for an overview of the model.

The authors were able to score 650,000 variants and found that the disease-causing variants tend to have higher scores than common variants [54]. They also found that for DNA variants that are 30 nucleotides away or more from a splicing site, the computational model predicted that known-disease variants are nine times more likely to affect the splicing process of mRNA when compared to normal variants [54].

The ability to predict DNA variants that cause splice events and are linked to diseases, allows the scientific community to identify and better understand the functional differences among protein isoforms that arise from the affected mRNA transcripts. 


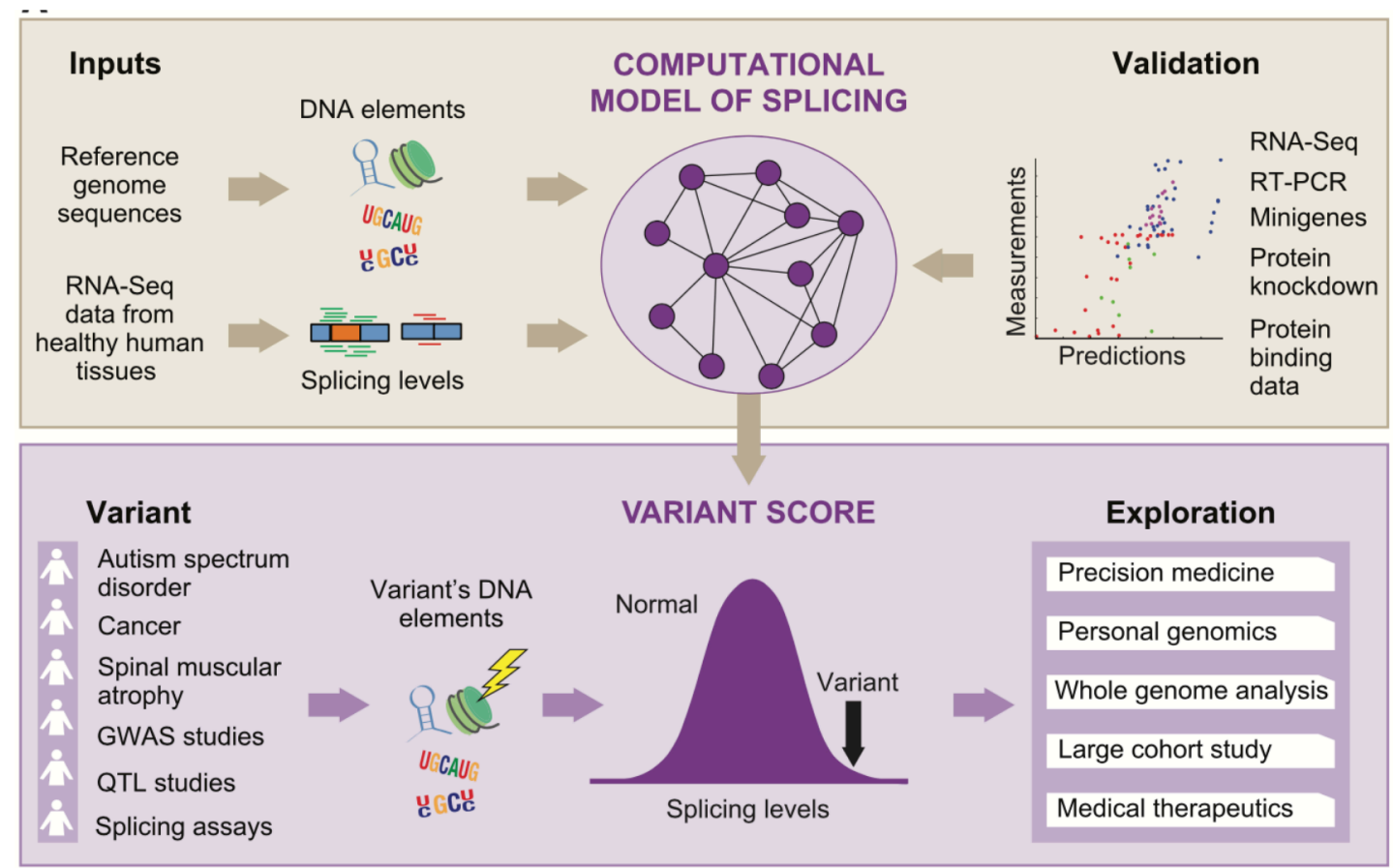

Figure 2.5: Top: Machine Learning is used to infer a computational model of splicing, by correlating DNA elements with splicing levels in healthy human tissues. Bottom: genetic variants arising from a wide array of diseases and technologies can be detected and filtered using the computational model, enabling explorations into the genetics of disease [54]

\section{Domain-Based Prediction of the Human Isoform Interactome}

Another method that attempted to solve the problem of assigning functions at the isoform level is called Domain-based Isoform Interactome Prediction (DIIP) introduced by Ghadie, Lambourne, Vidal \& Xia [12]. DIIP is a computational method that uses Domain-Domain interaction data along with datasets of experimentally validated pairs of interactions to infer the isoform-isoform interactome. 
11,557 Domain-Domain interaction reference records were obtained from the 3did database and from the DOMINE database of protein domain interactions [12]. The experimentally validated binary interactomes that were used in their study come from two datasets, HI-II-14 and IntAct. The HI-II-14 dataset contained 901 binary interactions among 917 reference proteins that contain annotated domain-domain interactions. The IntAct dataset on the other hand had 4,363 domain mediated interaction pairs from 2,944 reference proteins. For the 917 reference proteins from the HI-II-14 dataset, there were 1,227 alternatively spliced isoforms that were considered for this study [12]. Similarly, for the IntAct dataset, there were 4,471 alternatively spliced isoforms that came from the same genes as the 2,944 reference proteins. See Figure 2.6 for an overview of the study pipeline.

The authors first constructed a reference proteins interaction network by using experimentally determined protein-protein interactions and then each of these interactions were annotated by the domain-domain interactome. Subsequently, they constructed the isoform-isoform interaction network such that the alternatively spliced isoforms will inherit the interaction network of the main isoform (the canonical isoform) depending on whether the interaction mediating domains exist in the alternatively spliced isoforms [12]. An interaction of an alternatively spliced isoform is predicted to be lost only if all annotated domains that participate in the interaction are lost.

The researchers found that $22 \%$ of genes that have two or more isoforms have at least one isoform that lost an interaction compared to the canonical isoform [12]. They also found that from a total of 16,848 ASI interaction predictions made across both HI-II-14 and IntAct datasets, the computational model predicted 14,836 ASIs to have retained their interactions compared to 2,012 ASIs that have lost interactions relative to their reference proteins [12]. 


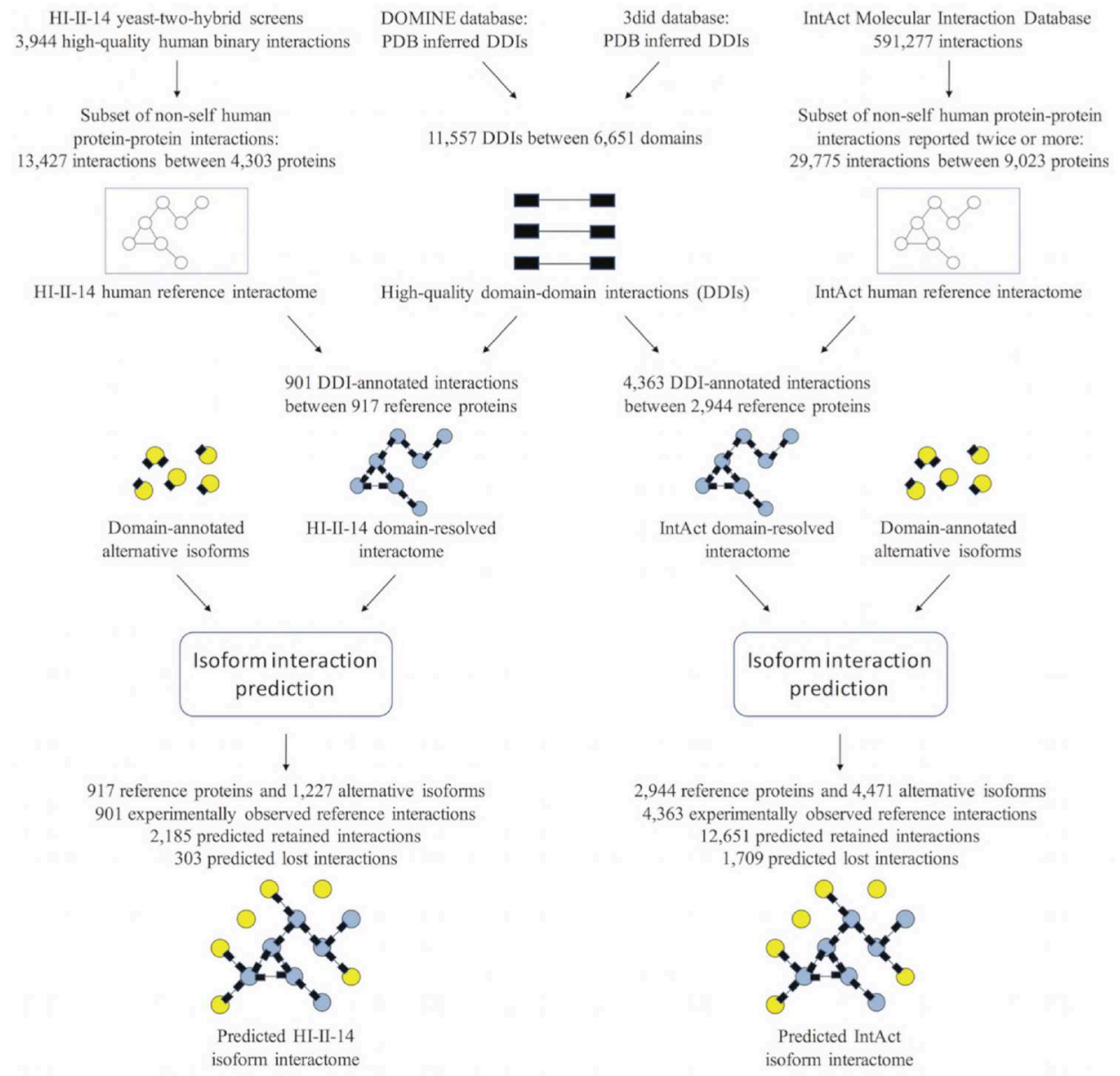

Figure 2.6: Pipeline for the DIIP algorithm [12]

When comparing isoform interaction profiles, the authors assigned the pair of proteins to be compared into one of three groups: pairs of proteins interacting with the same subset of the isoforms of the same gene, pairs of proteins interacting with different subsets of isoforms of the same gene, or pairs of proteins interacting with protein products from different genes, refer to Figure 2.7 for an illustrative diagram of the three groups. 

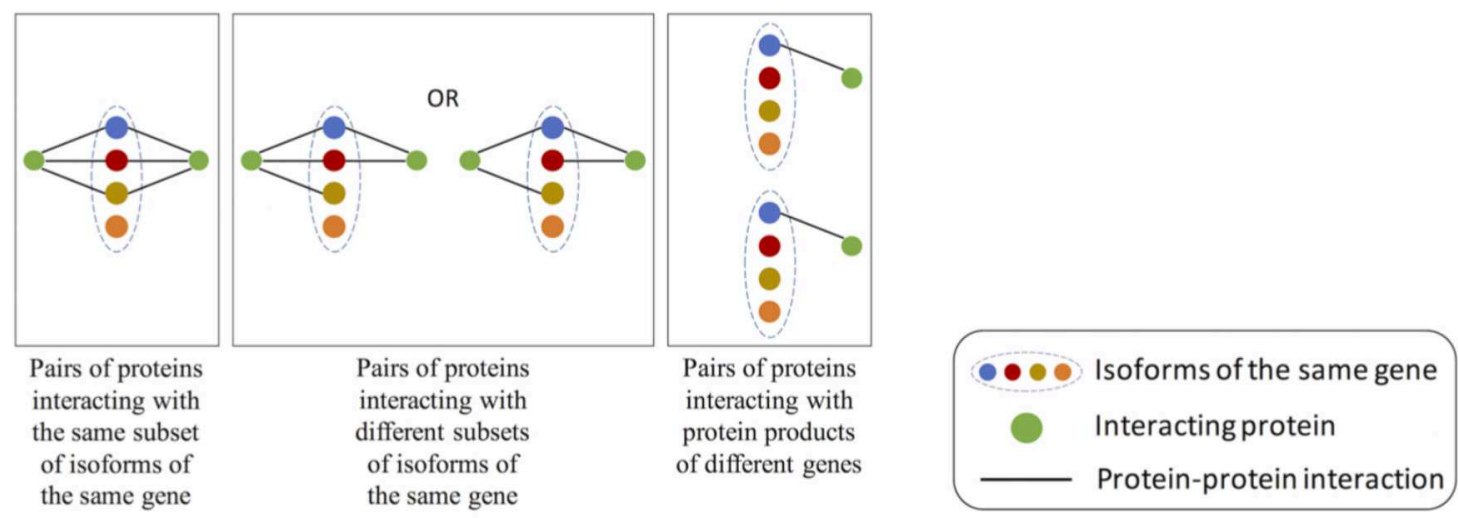

Figure 2.7: An illustration of different types of isoform interaction profiles for a given pair of proteins [12]

To compare isoform interaction profiles from the predicted isoform interactome, the authors used reference proteins that have Gene Ontology annotations as a base for comparisons. They then used the Jaccard similarity index to calculate GO profile similarity scores between protein pairs [12].

They found that, on average, protein pairs that fall under the first group (pairs of proteins interacting with the same subset of the isoforms of the same gene), have a significantly higher GO similarity scores in all three GO categories (Molecular function, Cellular component, and Biological process) than the other two comparison groups [12]. This shows that AS can result in loss of interactions that in turn can result in functional differences in the human proteome.

The authors proceeded to use disease annotations and expression profiles of the reference proteins they have in their study to compare the three comparison groups 
of the predicted isoform interactome. They found that the less similar the protein pairs being compared were in terms of expression levels and disease profiles, the more different the predicted interaction profiles of their results were [12].

Although the computational model in this study only considers losses of isoform interactions compared to the reference proteins, the authors were able to demonstrate that alternative splicing of mRNA produces divergent interaction networks that could give rise to different functions among isoforms that belong to the same gene.

\subsection{RNA-seq Non-Computational Analysis Approaches}

A number of studies rely on RNA-seq data without a computational model that focuses on alternative splicing and its effects on functional diversity in eukaryotes. These studies are largely based on RNA-seq expression data, which allowed for the measurement of expression levels of alternative ORFs in various tissue types and under different conditions. This in turn makes it possible to conduct comparative studies of protein isoforms and potentially labeling functions or establishing disease links to isoforms according to their expression profiles. In the following section, we review two of these studies.

\subsubsection{Yeast-two-Hybrid Study}

Yang et al. conducted a study on the effects of alternative splicing in humans where the authors cloned open reading frames for alternatively spliced isoforms coming from a set of human genes and conducted a Protein-Protein interaction profiling experiment to functionally compare isoforms [56]. 
The authors study included ORFs coming from $8 \%$ of the human protein-coding genome that were extracted from different human tissues and have also well-studied Protein-Protein interactions [56]. The authors were able to recover 1423 ORFs 917 of which were alternatively spliced ORFs. The remaining 506 ORFs were identified as reference ORFs.

The authors proceeded to compare the recovered alt-ORFs and reference ORFs in terms of expression levels in four different types of tissues. They found that almost half of the genes recovered have their alt-ORF expressed more than its reference ORF in at least one tissue [56].

For their Protein-Protein interaction comparisons, the authors used a Yeast twohybrid (Y2H) screen of a total of 1035 protein isoforms from their set. They obtained results for 366 isoforms, 248 of which had one or more confirmed binary interactions resulting in a total of 1043 PPIs with 381 proteins [56]. Review Figure 2.8.

To better understand the interaction behavior of isoforms, the authors identified isoform-specific regions (ISRs). To be identified as an ISR, a region of an ORF has to be at least 40 amino acids long, and is present in one, a subset, or in all isoforms of genes being tested [56]. Subsequently, isoform interaction protein partners resulting from the $\mathrm{Y} 2 \mathrm{H}$ screen were categorized into four categories promoting, inhibiting, promoting or inhibiting, and complex. Promoting partners indicate that the protein interacts exclusively with isoforms that contain a certain ISR. Inhibiting proteins are those that do not interact with isoforms that contain a given ISR. Promoting or inhibiting partners are proteins that are involved in interactions that have a given ISR and does not have another ISR. Complex partners are partners that do not belong to any of the previous groups [56]. 


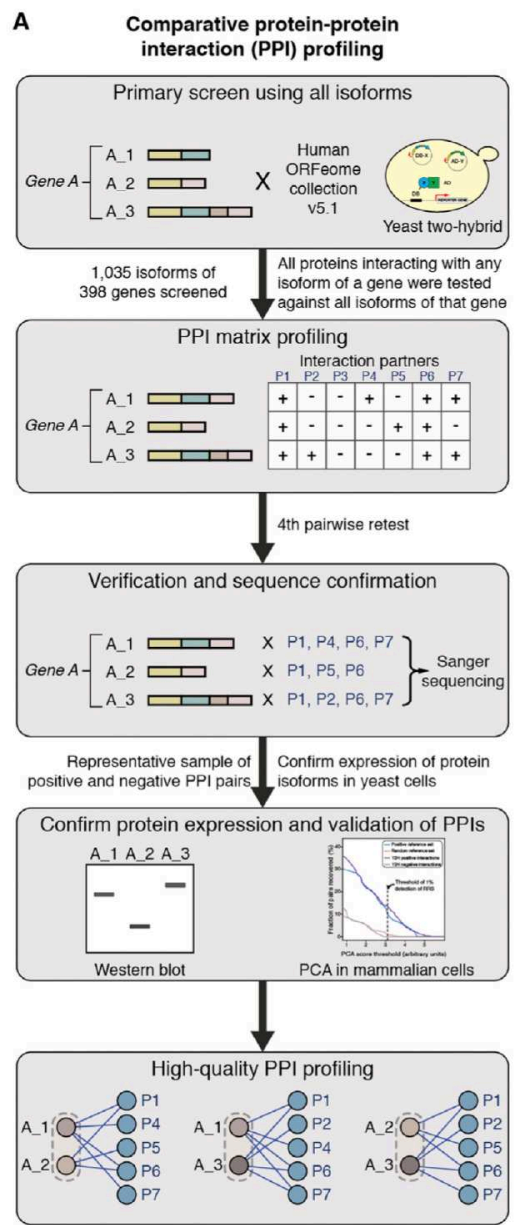

Figure 2.8: Process for comparative protein-protein interaction profiling $[56]$

When annotating Linear Motif Binding Domains (LMBD) sites obtained from the Eukaryotic Linear Motif resource (ELM) onto isoforms, it was found that linear motifs presence was more abundant in isoforms that participated in interactions containing promoting partners than those that were participating in interactions containing inhibiting partners (validated by using two-sided Wilcoxon rank sum test, $\mathrm{p}=$ $0.005)[56]$. 
The authors also studied Domain-Domain interactions in the context of their results. They found that out of 60 non-canonical isoforms that lost 50 or more domainlinked residues, 52 also lost protein interaction partners when compared to the canonical.

Finally, the authors used the Jaccard similarity index to measure the differences in interaction profiles between alternatively spliced isoforms that come from the same gene. According to the Jacquard index, 1 means that the compared isoforms have identical protein interaction profiles while 0 indicates a unique interaction profile for both isoforms. They found that $16 \%$ of comparisons yielded an index of $0,21 \%$ had an index of 1 , and $63 \%$ had a index between 0 and 1 .

This study yielded results that suggest that alternative splicing introduces differences at the functional level inferred from the differences found among the interaction profiles of isoforms belonging to the same gene.

\subsubsection{ISOexpresso, alternative splicing, and cancer}

The authors of this study designed a tool that analyzes the differential expression profiles of protein isoforms in 30 cancer tissues [55]. Using RNA-seq data coupled with clinical information obtained from The Cancer Genome Atlas (TCGA), the model was designed to be able to produce comparative expression levels of tumor vs normal conditions of protein isoforms of a gene of interest in different types of human cancer tissues. See Figure 2.9 for an outline of the tool [55].

The data obtained from TCGA included 9,499 tumor samples and 735 normal samples along with their RNA-seq expression data for 30 cancer tissues [55]. For the comparative normal vs tumor analysis, only cases which contained more than 10 normal and 


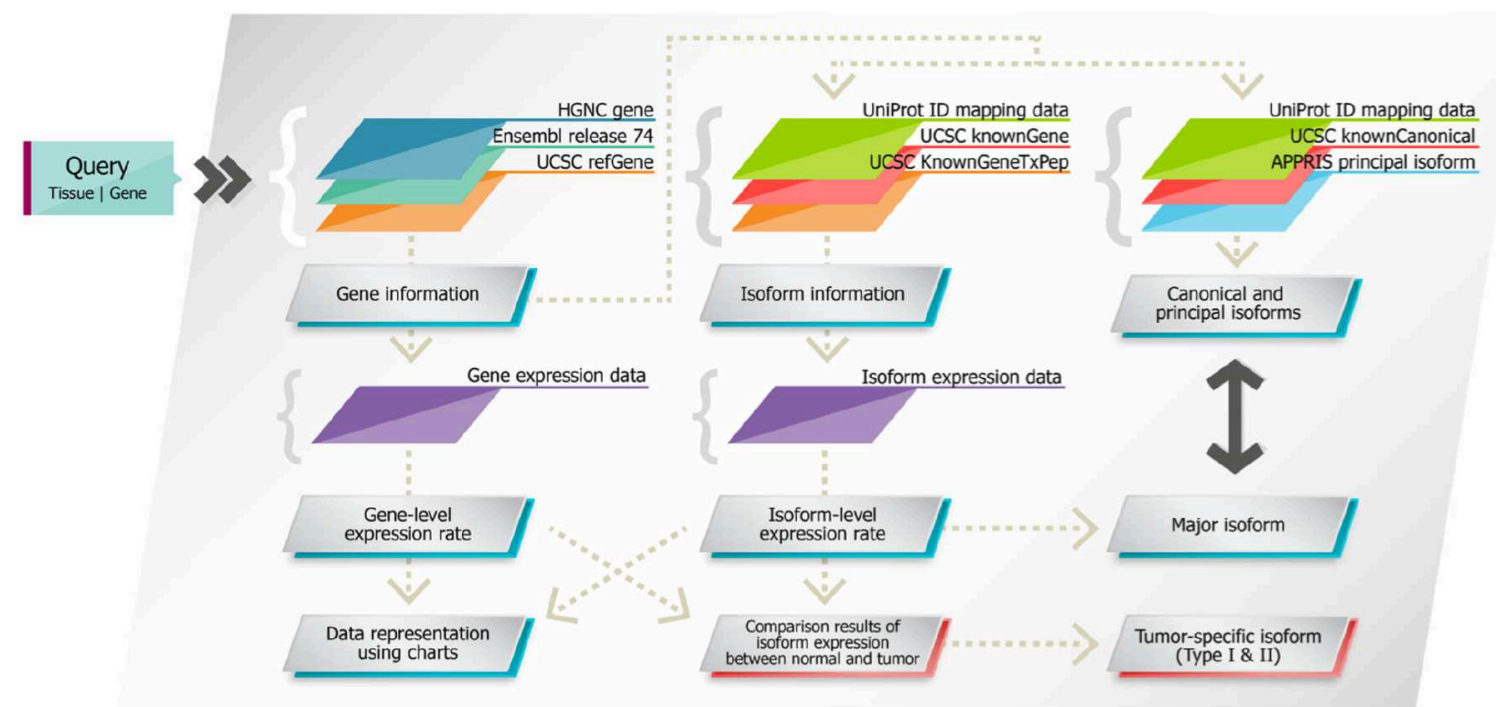

Figure 2.9: General overview of ISOexpresso workflow [55]

more than 10 tumor tissues were considered in the analysis. The study covered 26,541 human genes; however, only 17,532 genes had expression data [55]. The authors also designed a web tool that allows users from the public to search for a gene of interest by showing its isoforms expressions in normal vs tumor conditions.

They found that in tumor and normal tissues, the canonical isoform was expressed more than other isoforms of the same gene in $70 \%$ of the cases. However, in the $30 \%$ of cases where a non-canonical isoform was expressed more, they found that these cases were mostly in tumor tissues [55].

The authors used three genes that are confirmed to have non-canonical isoforms expressed higher than the canonical isoforms as validation examples of their tool accuracy. CD47 is a gene that has a non-canonical isoform that is known to be expressed more than the canonical in 16 tissues. The tool was able to discover 10 of the 16 and an additional 5 that were not known before [55]. FBLN2 is a gene that is 
known to be related to tumor-suppression and studies have reported that there are differential expression levels among its isoforms during tumorigenesis. ISOexpresso showed two of the non-canonical isoforms being expressed more than the canonical in nine cancer tissues [55]. Finally, CD44 is known to have variant expression levels for its isoforms which the tool reported upon query. Refer to Figure 2.10 for the results. 
a

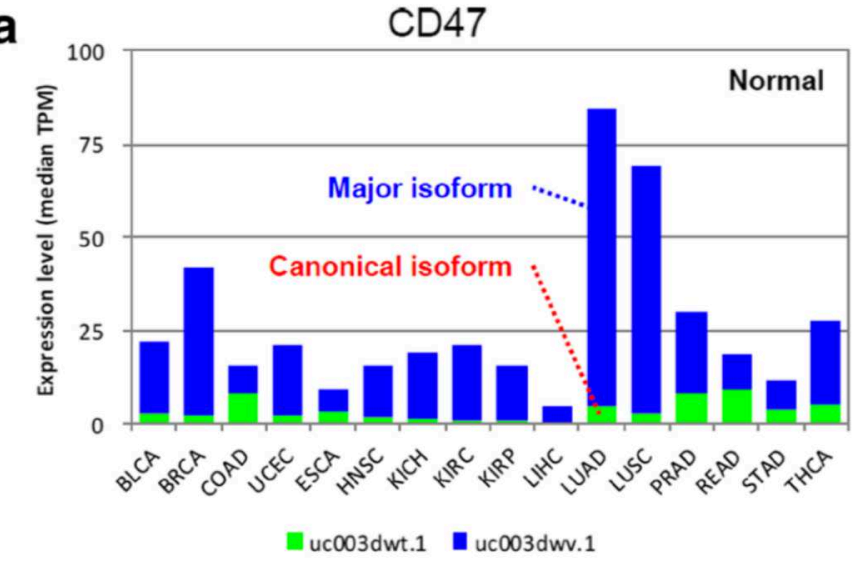

b

FBLN2

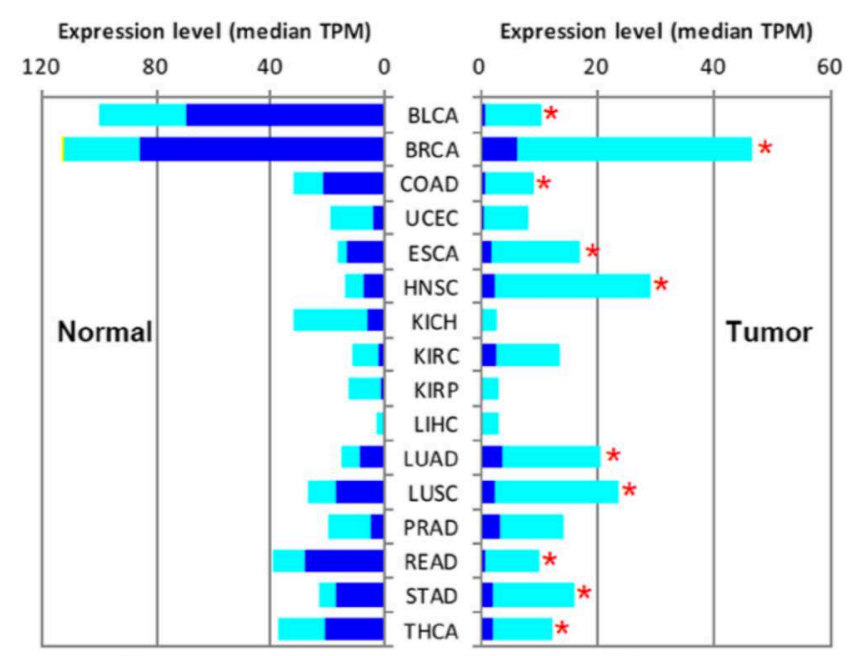

uc011auz.2 uc011ava.2 uc011avb.2 uc011avc.2

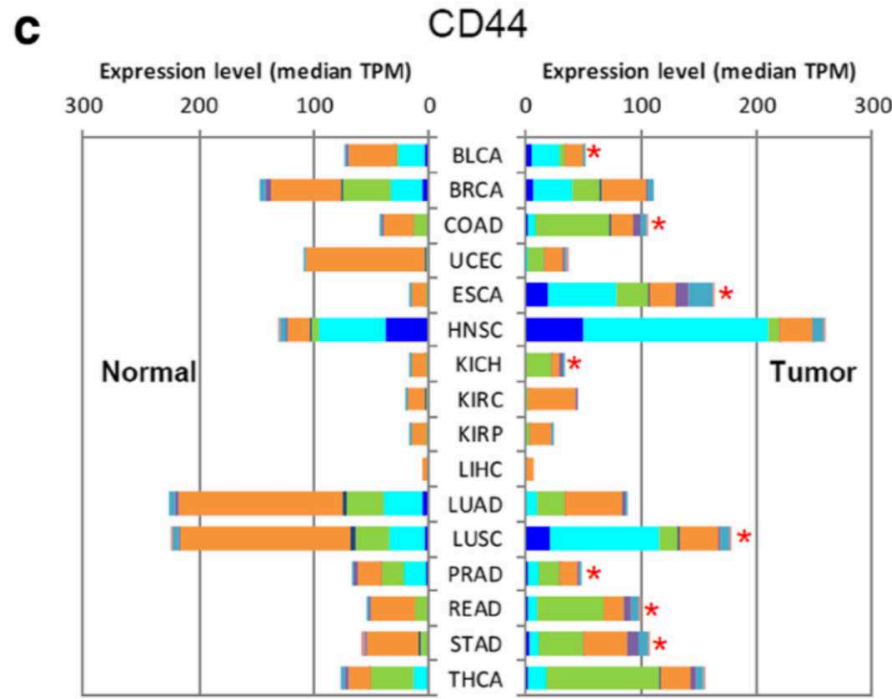

uc021qfw.1 घuc001mvu.3 uc uc001mvv.3 uc $001 \mathrm{mvw} .3$

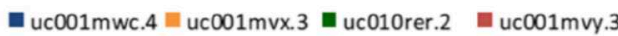

घuc009ykh.3 muc010reu.2 u uc010res.2 uc010ret.2

Figure 2.10: Three examples of expression levels for isoforms of genes of interest [55] 
The results found by this study led the authors to believe that alternatively spliced isoforms expression could be linked to disease proliferation, specifically cancer. There is a growing body of research that supports this claim.

\subsection{PIPE Algorithm Review}

The Protein-Protein Interaction Prediction Engine, otherwise known as PIPE, is a computational method that predicts the likelihood of an interaction between two proteins based on their primary amino acid sequence. The method was developed at Carleton University by Pitre et al. [40]. By supplying the algorithm with an experimentally validated set of PPIs alongside their primary sequences, PIPE trains a model that can accurately predict interactions between two or more proteins.

The PIPE algorithm has been demonstrated to be an effective predictor of proteinprotein interactions by an independent study [57]. The original PIPE algorithm was able to predict the likelihood of an interaction between any two saccharomyces (yeast) proteins with an accuracy of 75\% [40]. Many major improvements and refinements have been implemented since then that allowed the algorithm to achieve unprecedented feats such as the computation of the PPIs of the whole human proteome.

PIPE takes two elements of information as input, a list of protein pairs that are known to interact, and query proteins along with their amino acid sequences (proteins that we want to know whether they interact). PIPE converts the list of known interacting pairs into a graph $G$ where nodes are proteins and edges are interactions. PIPE would then start comparing proteins as follows: Given a protein $A$ that is being compared to a protein $B$, PIPE would start comparing protein $A$ 's sequence to every protein 
in graph $G$ using a sliding window of amino acids of size $w(0 \leq w<|A|)$. The initial window in protein $A$ covers the first $w$ amino acids and would be compared to a similar window in protein $X$ from graph $G$. If the two windows match, all the proteins that are connected by an edge to $X$ are added to a list $R$ (interacting partners of $X$ ). Matches are determined by using the PAM120 scoring matrix. After the comparison is done, the window shifts by one amino acid to the right in $X$ and another comparison is made. This process continues until $X$ is fully compared to the first window in $A$ after which the window in $A$ shifts by one amino acid to the right and the same process entails until all windows in $A$ are compared to all windows in $X$. $A$ is then compared to all proteins in $G$ in a similar fashion [40].

After PIPE is done all the comparisons with $A$, protein $B$ would now be compared to all the proteins in $R$ in the same way protein $A$ was compared to proteins in $G$ [40]. When a matching window in $B$ is compared to a matching window in protein $V$, a matrix of length $|A|$ and width $|B|$ would be incremented by 1 in cell $(\mathrm{i}, \mathrm{j})$ where $\mathrm{j}$ is the window of protein $B$ that matched $V$ and $\mathrm{i}$ is the window of $A$ that matched $X$ which is also an interacting partner of $V$. After the whole process is done, a 2D matrix is produced by PIPE that shows how frequent windows of both protein $A$ and $B$ co-occur across all comparisons. The more frequent the overlap between the two proteins, the more likely that proteins $A$ and $B$ interact. The PIPE score is then calculated for each interaction pair as the average of all cell values across the produced matrix. In addition, PIPE has an alternative scoring method called the similarity weighted score. Although the sim-weighted score leverages the same mechanics as the traditional PIPE score, it also takes into account the frequency of motifs being considered (represented by the sliding window). The more a motif occurs in other proteins, 
the less weight it carries in the final prediction score. Thus, the sim-weighted score normalizes the final score based the uniqueness of interaction mediating motifs [39]. Figure 2.11 shows an overview of the whole process [40].

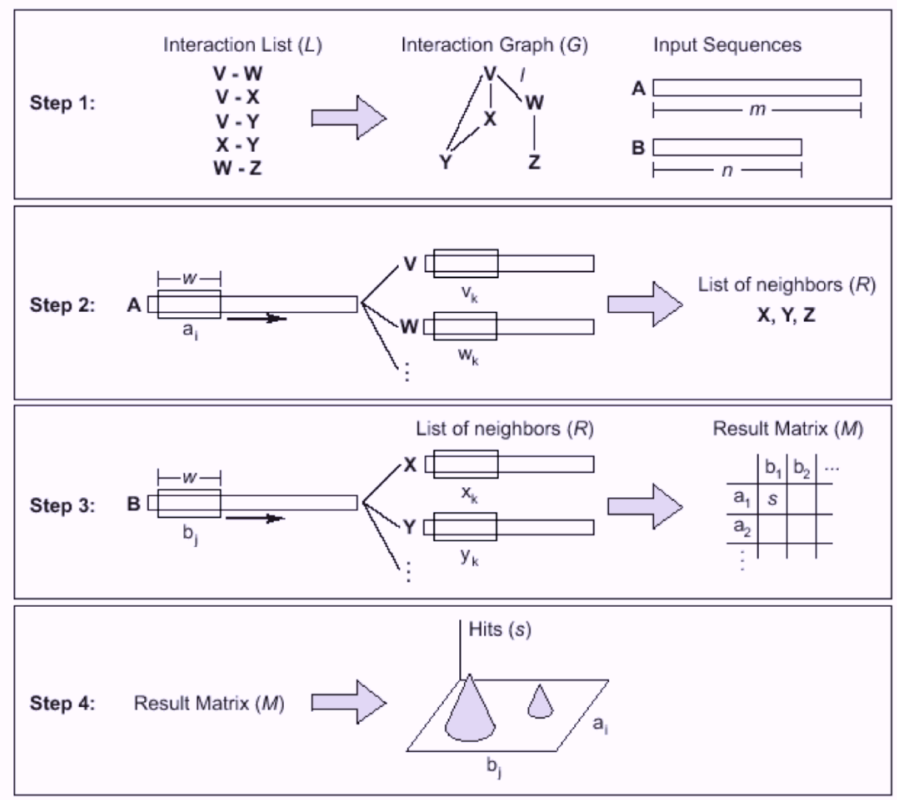

\section{String comparison}

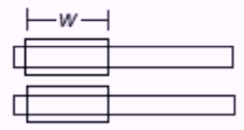

PAM 120

$\begin{array}{lllllll}A & R & N & D & C & \ldots\end{array}$

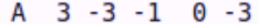

$\begin{array}{llllll}\mathrm{R} & -3 & 6 & -1 & -3 & -4\end{array}$

N

D $\begin{array}{llllll}0 & -3 & 2 & 5 & -7\end{array}$

C $\begin{array}{llllll}-3 & -4 & -5 & -7 & 9\end{array}$

$\cdots$

Match $=$ (Sum of pairwise PAM values > Threshold)

Figure 2.11: Steps taken by PIPE when comparing the sequences of two proteins $A$ and $B[40]$ 


\section{Chapter 3}

\section{Alternative Splicing Effects Analysis}

\subsection{Pipeline Overview}

In this section, the work of this thesis will be explored. The main goal of this study is to provide the research community with a tool that sheds light on the degree to which AS affects the human proteome in terms of cellular function, tissue expression, and relationship to diseases. In the upcoming sections, the results of analyzing a subset of the human proteome for the effects of AS will be reviewed along with the procedures and the tools used to reach the results through an analysis pipeline. Before starting the analysis pipeline, we will take a step back and conduct a high-

level study of the AS isoform sequences' dataset we are examining. For this study, a 
canonical isoform is defined based on the Uniprot database definition of a canonical which corresponds to the most prevalent isoform, or the longest isoform when isoform data is absent [1]

The AS dataset study aims to gain insights of general physical differences between a CI and its ASIs amino-acid sequences. The analysis will shed light on the degree of similarity between ASIs and CIs sequences, the types of sequence changes that AS isoforms undergo, and subcellular localization information of genes that undergo AS.

The analysis pipeline includes multiple steps that transforms the raw data of primary amino acid sequences into functionally labeled interaction networks with annotated tissue expression profiles.

The pipeline starts by training a machine learning model that predicts the interaction partners of a protein. We will be using PIPE to predict the interaction partners for each protein isoform (CIs and ASIs). These IPs will then makeup the PPI networks for each of the protein isoforms that are subsequently used to infer PPI differences between CIs and their ASIs. At the end of this step, we produced a ranked list of ASIs based on the degree of changes between their PPI network and their CI's PPI network.

To be able to differentiate between CIs and ASIs from a functional perspective, a GO-Term labeling tool called GO-Term Finder is then used to functionally annotate each of the predicted PPI networks. By supplying PPI networks as input, the tool is capable of inferring GO annotations that the PPI network IPs are likely to share. This information is then used to determine which functions were gained or lost between a CI and an ASI PPI networks. 
Additionally, the pipeline includes a step in which protein isoform expression data from multiple tissue types is used to analyze ASIs expression patterns in 32 tissue types and compare/contrast them to the their relative CIs. This allows for the exploration of the possible effects that AS can have on tissue expression.

In the subsequent pipeline step, the results from the CIs and ASIs PPI construction step and the results from the tissue expression changes analysis step are used to complement each other by overlapping ASIs with significant changes/differences from both result sets. This is done by first cross-linking isoforms from both result sets by their UniProt-ID and then finding ASIs that have significant PPI network changes as well as significant tissue expression changes relative to their CIs.

Finally, we will review the pipeline results for the top ten overlapped ASIs from the previous step in conjunction with published studies and communicate our findings.

See Figure 3.1 for an overview of the analysis pipeline. 


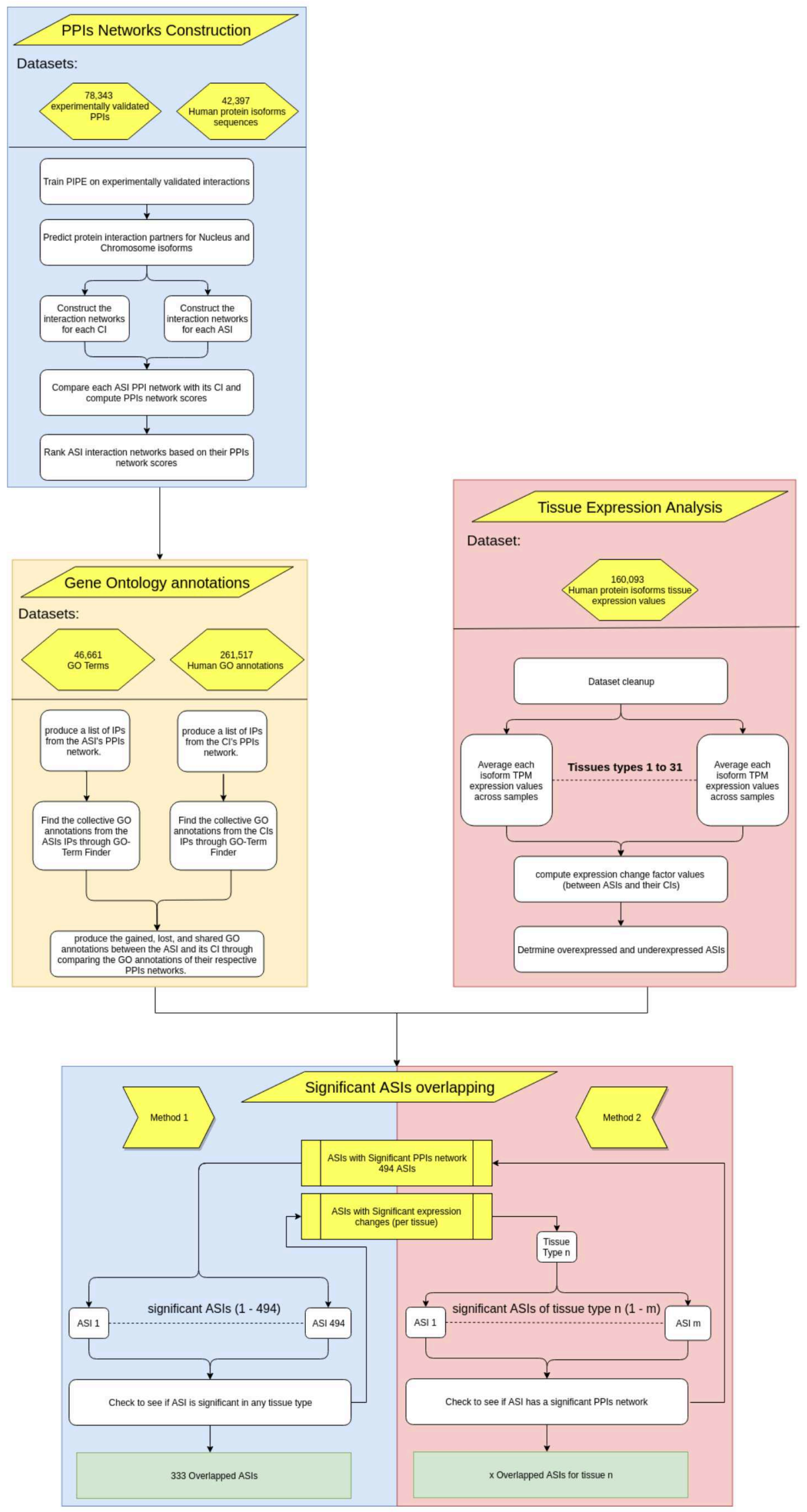

Figure 3.1: Analysis pipeline overview 


\subsection{Datasets collection \& cleanup}

The analysis in this study is performed on four human datasets including the general proteome dataset, the protein isoform sequences dataset, the protein isoform tissue expression dataset, and the Gene Ontology annotations definition dataset. In this section we explain the processing steps of the first two datasets while the latter two will be explained in their upcoming related sections.

The general proteome dataset was fetched from the Uniprot consortium as a downloadable XML file and contains the general information of the human genes, and their related proteins [1]. The data in the dataset includes gene names, functions, cellular localizations, protein sequences, GO annotations, and other gene-related properties.

Python scripts and libraries were used to parse and extract the contents of the XML file and store them in an SQLite database. Any gene that had missing critical information would be labeled as such and would not be considered in later steps. At a minimum, a gene has to have the following to be considered:

- A Gene symbol represented by a string of alphanumeric characters.

- At least one protein-coding sequence.

- That the gene has been manually reviewed by researchers. Uniprot has a label 'Reviewed' that is given to manually curated genes.

Subsequently, the protein isoform sequences dataset was parsed. This dataset was fetched from the Uniprot consortium in a FASTA format [1]. It contains all the 
recorded alternatively spliced isoforms of human genes. Similar to the first dataset, this set was parsed by a Python script that added protein isoform sequences to the SQLite database based on the genes they arise from.

Ultimately, both datasets produced the 42,397 canonical and alternatively spliced isoform sequences (from 20,098 human coding genes) that were considered in subsequent pipeline steps.

\subsection{Dataset Analysis}

Upon analyzing the dataset, it was found that alternative splicing occurs in genes localized in different sub-cellular locations with varying degrees. Figure 3.2 outlines the top five sub-cellular locations in which genes that undergo the process of alternative splicing are present. The cytoplasm, the nucleus, and the mitochondria have more than half of all the genes that undergo AS in the human genome. Also, it was found that alternative splicing affects genes with varying degrees where some genes have ten or more alternatively spliced isoforms while others have only one alternatively spliced isoform. Figure 3.3 shows the distribution of alternatively spliced isoforms per gene. 


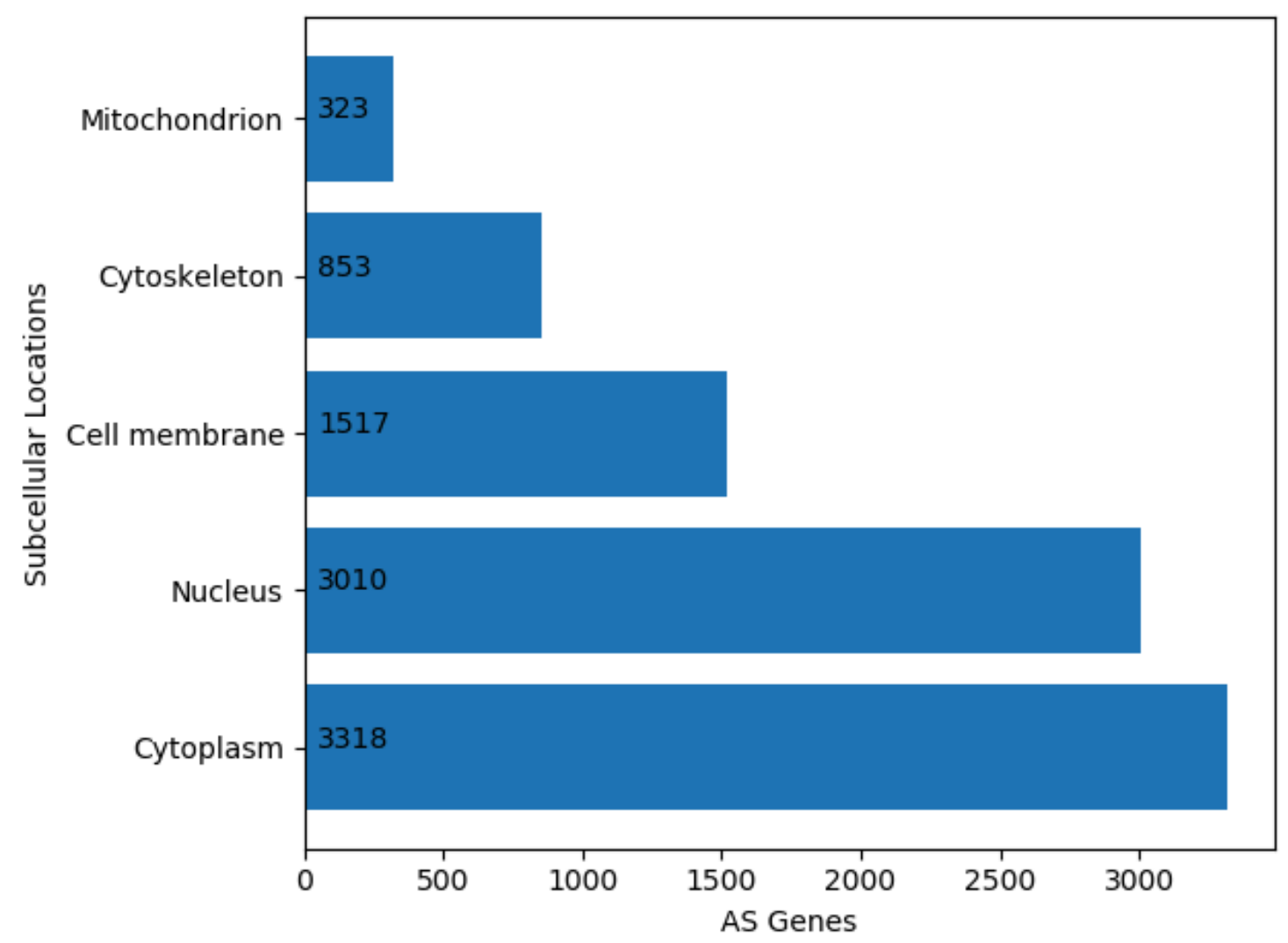

Figure 3.2: Highest five human subcellular locations in terms of number of annotated AS genes

The distribution of ASIs per gene has an interesting pattern. By reviewing Figure 3.3, it can be noticed that there is a strong negative correlation between the frequency of genes and the number of ASIs they have. Genes that have one alternative isoform are the most frequent followed by genes that have two isoforms where the gene frequency roughly halves. This trend where the frequency of genes drops by half continues as the number of ASIs per gene increases. 


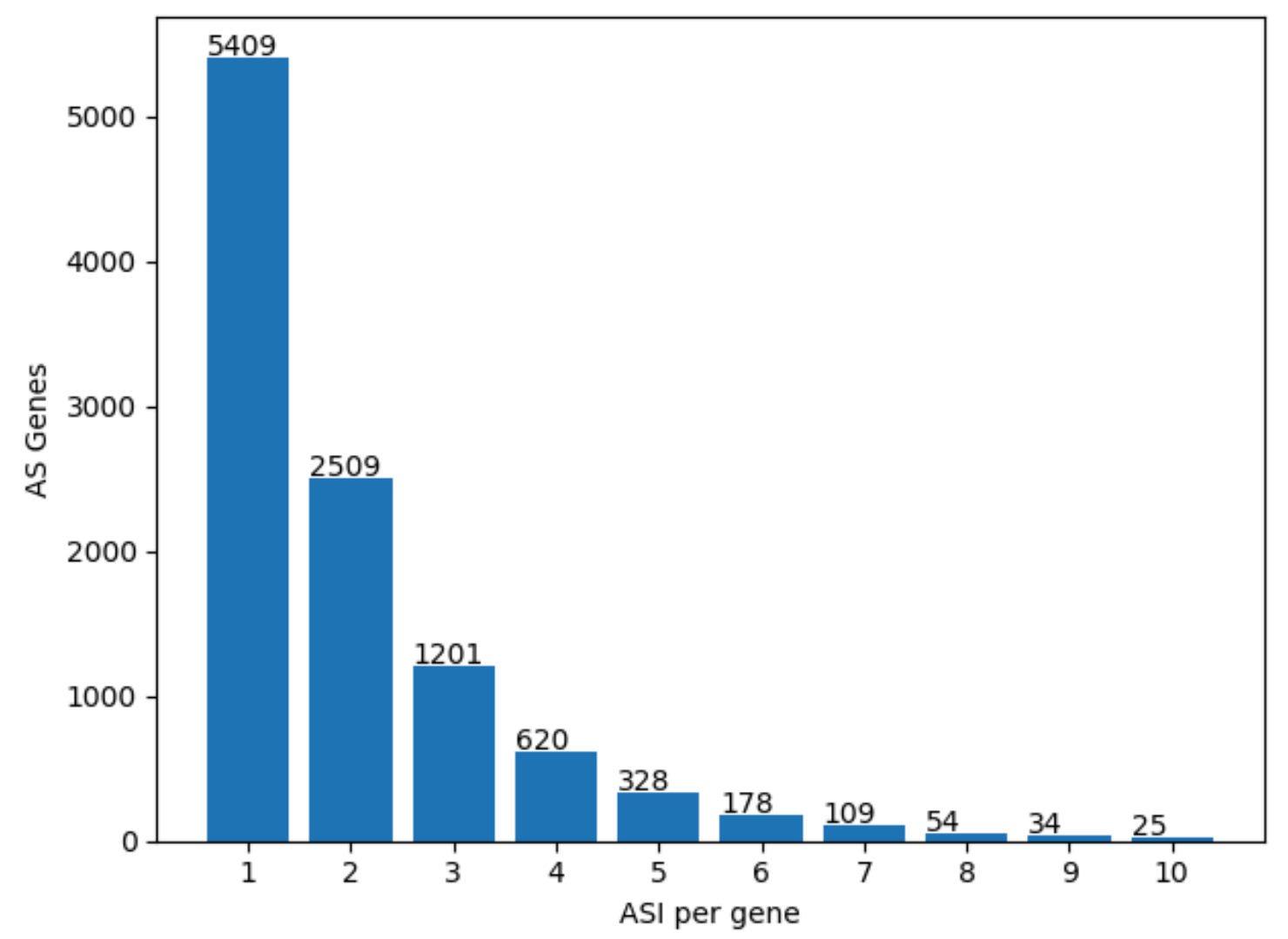

Figure 3.3: Frequencies of AS genes based on the number of alternatively spliced isoforms they have

Out of the 42,397 protein isoforms, 9,642 are non-AS canonical isoforms (canonical isoforms that do not have any ASIs), 10,556 AS canonical isoforms while 22,199 are alternatively spliced isoforms. This shows that AS occurs with a fairly large frequency in the human genome where more than half the human genes have alternatively spliced isoforms.

For this study, we focused only on ASIs that come from genes localized in the nucleus or the chromosome with a total of 9,331 isoforms (2,737 non-AS CIs, 2,327 AS CIs and 4,267 ASIs). See Figure 3.4 for more details about the dataset. The Nucleus 
and the Chromosome subset was chosen because of the small scale of this project as it was faster to construct the PIPE PPI networks, while also being a representative subset as can be noted from figures 3.4, 3.5, and 3.6.

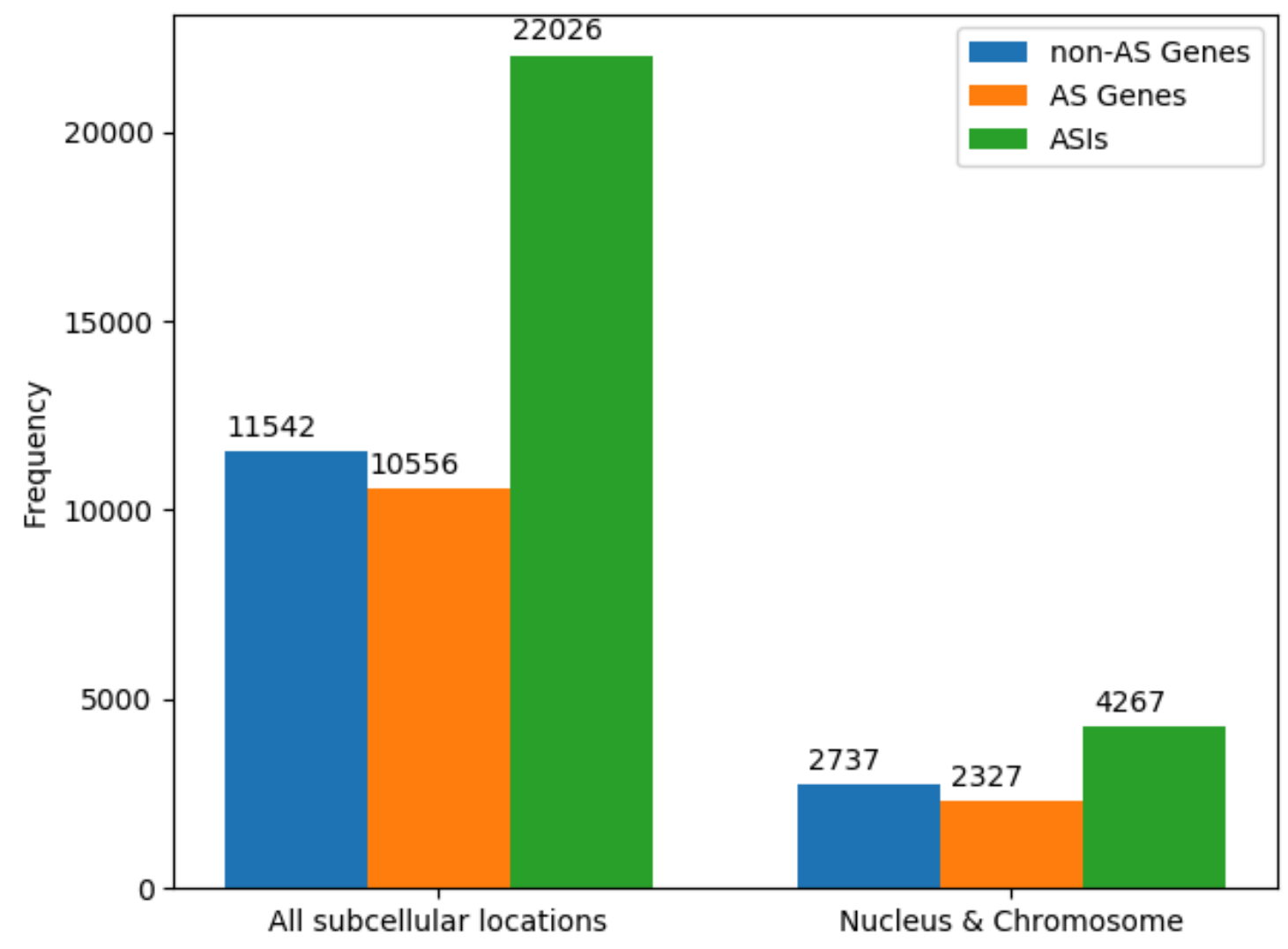

Figure 3.4: Comparison between the full human set and the nucleus and chromosome subset in terms of frequencies of non-AS genes, AS genes, and ASIs

After conducting a global sequence alignment comparison between the ASIs and their relative CIs, it was apparent that ASI sequences differ from their relative CIs sequences by either of two ways. ASI sequences either include (add) or exclude (trun- 
cate) amino acid sequences relative to the CI sequence. In some few cases, the CI and the ASI sequences are identical. See Figure 3.5 to view the percentages of each change type.

\section{All subcellular locations}

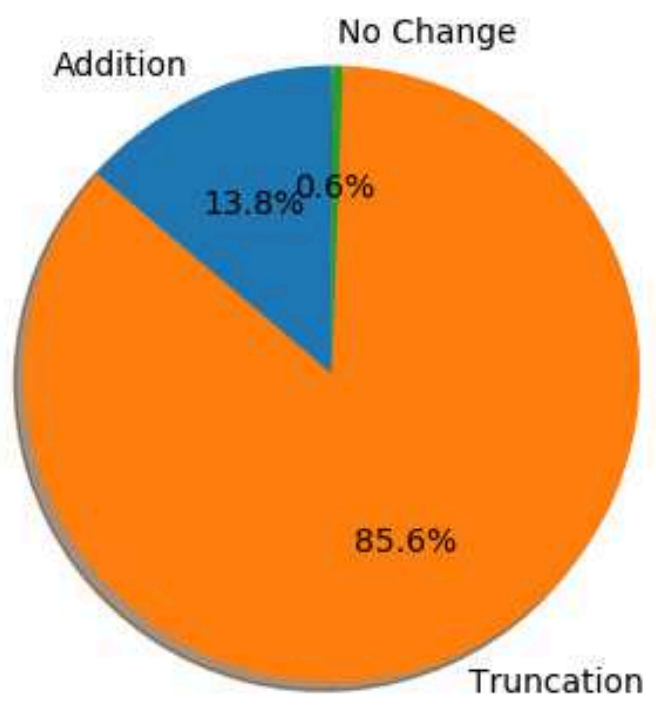

\section{Nucleus \& Chromosome}

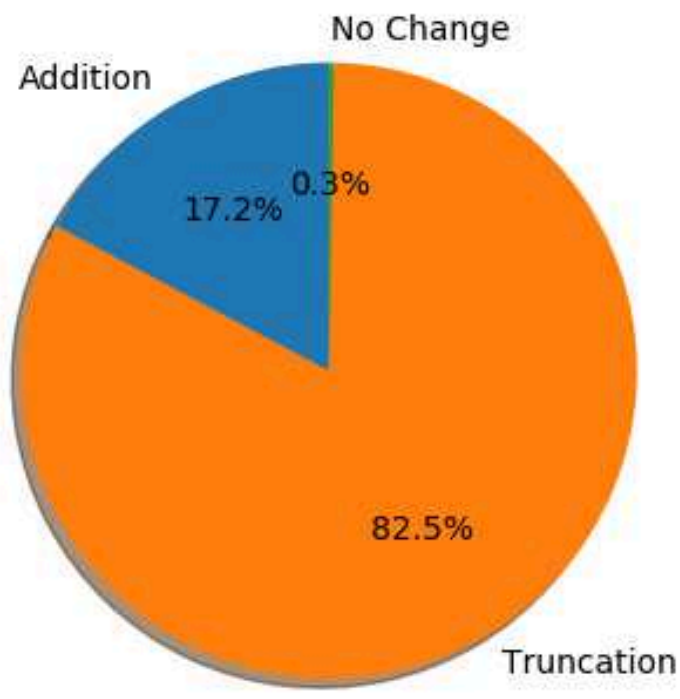

Figure 3.5: Comparison between the full human set and the nucleus and chromosome subset in terms of the types of sequence changes ASIs have relative to their CIs

The global alignment produces a score of similarity for each comparison made between an ASI and its CI. By aggregating the similarity scores from all the comparisons, it becomes clear that ASI sequences tend to be similar to their CIs. Refer to Figure 3.6 to view the aggregations of ASIs based on their similarity percentages. On average, 
ASIs have an isoform sequence similarity score of $75 \%$ relative to their CIs globally compared to $79 \%$ for the nucleus and chromosome set. This indicates that the degree of change that ASI sequences incur is conserved. Figure 3.5 illustrates that the majority of changes that the ASIs have relative to their CIs are sequence truncations making the ASI sequence shorter than its CI. In many cases this change renders the ASI sequence non-functional as a result of losing vital sequence regions.

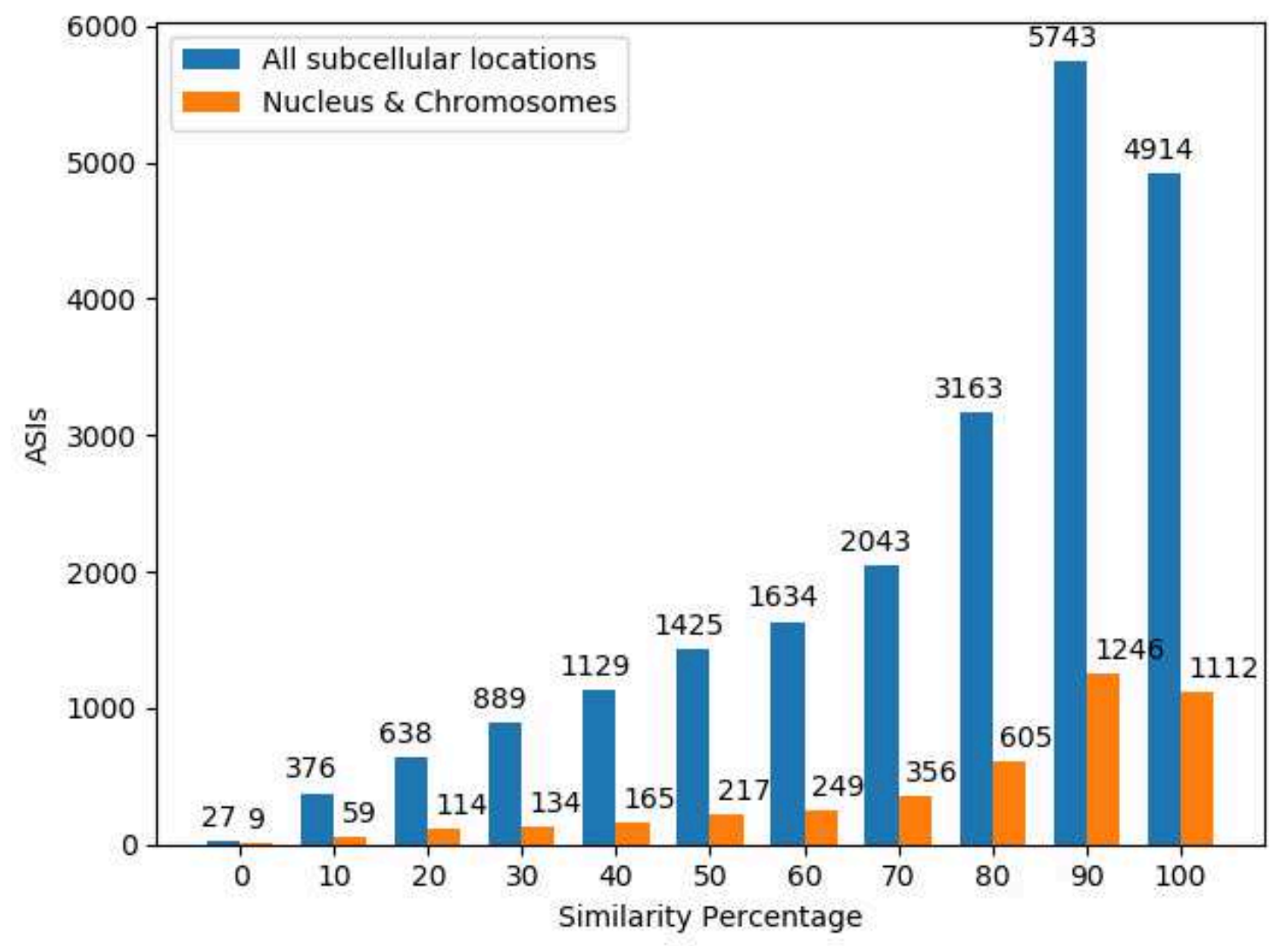

Figure 3.6: Comparison between the full human set and the nucleus and chromosome subset in terms of the sequence alignment similarity scores of ASIs relative to their CIs 


\subsection{Protein-Protein Interaction Networks Construc- tion}

\subsubsection{Introduction}

The first step of the analysis pipeline is where the PPI networks for both the CI and the ASIs from the AS dataset is constructed and compared. This step is the base step of the pipeline upon which all subsequent steps will build. The tasks in this section include the construction of PPI networks by predicting protein interactions using PIPE. Followed by the comparison of the predicted CIs and ASIs PPI networks, and finally concluded by the ranking of ASI PPI networks based on the computed AS PPI network score.

\subsubsection{PIPE Setup and Predictions of PPIs}

The Protein Interactions Prediction Engine (PIPE) was used to produce the proteinprotein interaction networks used in this experiment. PIPE was able to predict interaction pairs from the 9,311 protein sequences related to either the nucleus or the chromosome subcellular locations which resulted in 43.5 million predictions. PIPE has two scoring methods to measure the accuracy of the predictions, namely the PIPE score and the sim-weighted score. The sim-weighted score was used in this study because it produces less false positives compared to the traditional PIPE score $[39]$.

The PIPE model was trained using 78,343 experimentally confirmed positive interaction pairs. Subsequently, leave-one-out cross validation was used to determine the 
appropriate cutoff score to use to separate true positives from true negatives when predicting interaction pairs. Through out this study, predicted interactions with a \%99 specificity or higher (corresponds to a score of 7 on the sim-weighted scoring measure) were considered true positives.

\subsubsection{Comparing Alternatively Spliced Genes' Isoforms}

For each gene, the CI interaction network is compared with the interaction network of every other ASI of the same gene and the difference in the interaction profiles is determined as follows:

For a given CI interaction network, each of its interaction partners (IPs) is checked for co-occurrence in the interaction network of the ASI of the same gene and vice versa. One of three cases will arise as a result of the comparisons:

1. If a CI interaction partner does not co-occur in the ASI interaction network, then the interaction partner is considered "Lost" from the ASI PPI network.

2. If an ASI interaction partner does not co-occur in the CI interaction network, then the interaction partner is considered "Gained" in the ASI PPI network.

3. If the CI interaction partner appears in the two interaction networks, then the interaction partner is considered "Retained" among the two networks.

The more "Lost" or "Gained" IPs that are found for a particular comparison pair of a CI and an ASI networks, the more divergent the alternative splicing PPI network 
of the given ASI is. To measure the extent of difference between a CI and an ASI PPI networks we use the following equation to calculate an ASI's AS PPI network score:

(IPs Lost + IPs Gained) x average of score differences across all IP changes.

This equation is meant to quantify the magnitude of difference between PPI networks based on the IP differences and their related score changes. The first part of the equation (i.e. IPs Lost + IPs Gained) measures the number of interaction partners that are not shared among the interaction networks of the CI and the ASI being compared. The second part of the equation, (i.e. average of score differences) measures the magnitude of the difference between the CI and the ASI in terms of all significantly different interaction partners interaction scores. A score of 0 indicates that the ASI PPI network is identical to the CI's PPI network. Theoretically, there is no maximum value for the AS PPI network score, and no definition of a high or a low score. However, the AS ASI score allows for the ranking of ASI PPI networks based on how similar or different they are from their CI's PPI network.

The equation can be simplified as follows:

$$
A S I_{A} \text { AS PPI network score }=\sum_{i=1}^{n} \mid I P_{i} \text { score difference } \mid
$$

Where $n$ is the number of $A S I_{A}$ PPI network IPs that have significantly different scores compared to the CI PPI network. Significant in this context means that the PPI networks of the ASI and the CI differ by at least two IPs (gained or lost) based on the defined \%99 specificity cutoff. 


\subsubsection{Illustrative Example}

For example, assume we have a gene $e_{x}$ with a canonical isoform $C I_{x}$ and two alternatively spliced isoforms for the same gene $A S I_{A}$, and $A S I_{B} . C I_{x}$ has four interaction partners $I P_{1}, I P_{2}, I P_{3}$ and $I P_{4}$, and $A S I_{A}$ has also four interaction partners $I P_{1}$, $I P_{2}, I P_{4}$ and $I P_{5}$, while $A S I_{B}$ has only two interaction partners $I P_{5}$ and $I P_{6}$. See Figure 3.7 for the interaction networks illustrations.
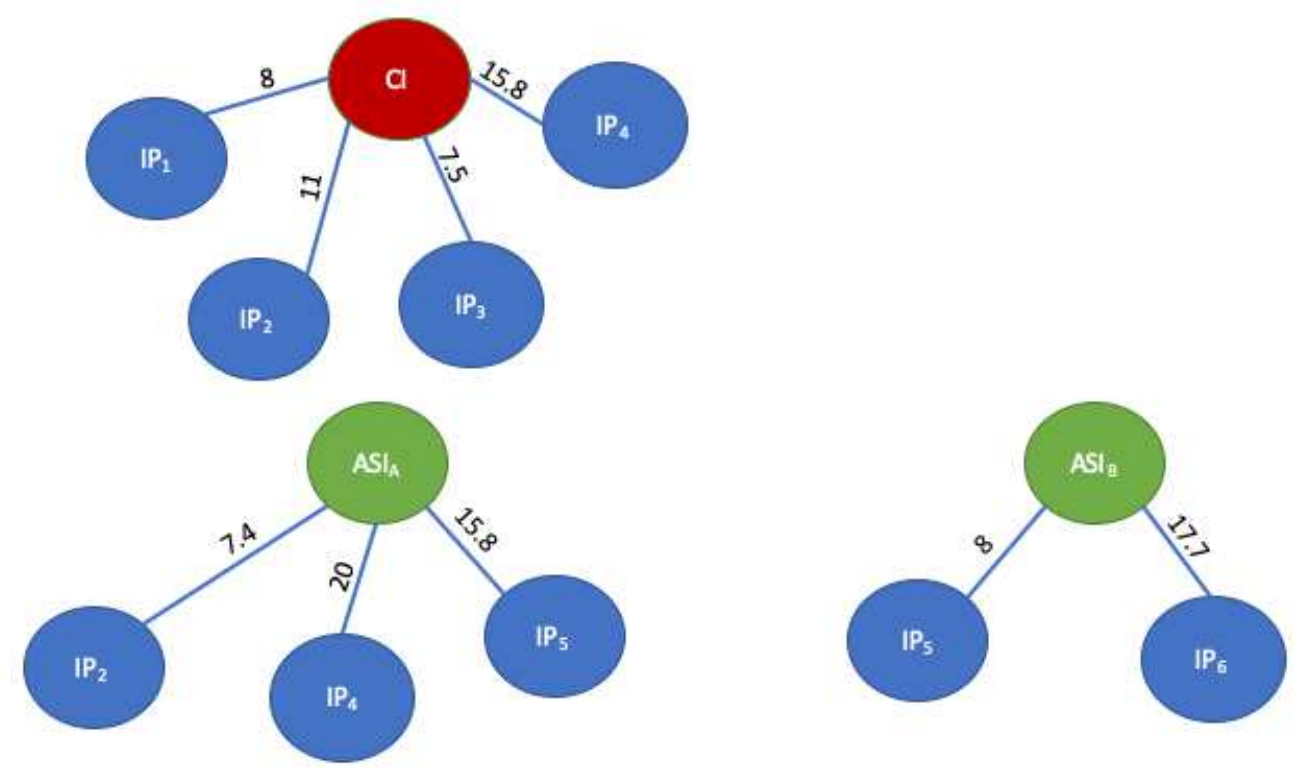

Figure 3.7: Illustrative example of constructed PPI networks

By using the \%99 specificity corresponding-cutoff, the comparison of the PPI network of the $C I_{x}$ with its $A S I_{A}$ will result in:

$I P_{1}$ (Lost), $I P_{2}$ (Retained), $I P_{3}$ (Lost), $I P_{4}$ (Retained) and $I P_{5}$ (Gained) See 3.1 for details about the $C I_{x}$ and $A S I_{A}$ comparison. 
The comparison of the PPI network of $C I_{x}$ with its $A S I_{B}$ will result in:

$I P_{1}$ (Lost), $I P_{2}$ (Lost), $I P_{3}$ (Lost), $I P_{4}$ (Lost), $I P_{5}$ (Gained) and $I P_{6}$ (Gained). See Table 3.2 for details about the $C I_{x}$ and $A S I_{B}$ comparison.

Finally, the $A S I_{A}$ AS PPI network score relative to $C I_{x}$ will be:

$A S I_{A}$ AS PPI network score $=6.5+13.8+5=25.3$

Similarly, $A S I_{B}$ AS PPI network score relative to $C I_{x}$ will be:

$A S I_{B}$ AS PPI network score $=6.6+10.7+3.2+12.8+13.8+4=51.1$

Based on the AS PPI network scores, we can conclude that $A S I_{B}$ has a more divergent alternative splicing PPI network than $A S I_{A}$.

\begin{tabular}{|l||l|l|l||l|}
\hline & $C I_{x}$ score & $A S I_{A}$ score & score difference & IP status relative to ASI \\
\hline$I P_{1}$ & 8 & 3 & -5 & Lost \\
\hline$I P_{2}$ & 11 & 7.4 & 3.6 & Retained \\
\hline$I P_{3}$ & 7.5 & 1 & -6.5 & Lost \\
\hline$I P_{4}$ & 15.8 & 20 & 4.2 & Retained \\
$I P_{5}$ & 2 & 15.8 & 13.8 & Gained \\
\hline
\end{tabular}

Table 3.1: $A S I_{A}$ and $C I_{x}$ PPI networks comparison 


\begin{tabular}{|l||l|l|l||l|}
\hline & $C I_{x}$ score & $A S I_{B}$ score & score difference & IP status relative to ASI \\
\hline$I P_{1}$ & 8 & 1.4 & -6.6 & Lost \\
$I P_{2}$ & 11 & 0.3 & -10.7 & Lost \\
$I P_{3}$ & 7.5 & 4.3 & -3.2 & Lost \\
$I P_{4}$ & 15.8 & 3 & -12.8 & Lost \\
$I P_{5}$ & 2 & 17.2 & 15.7 & Gained \\
$I P_{6}$ & 4 & 8 & 4 & Gained \\
\hline
\end{tabular}

Table 3.2: $A S I_{B}$ and $C I_{x}$ PPI networks comparison

\subsubsection{Results \& Discussion}

The previously mentioned criteria for constructing interaction networks and comparing AS PPI networks (i.e. \%99 specificity cut-off score) produced 494 ASIs that had significant PPI networks when compared to their CIs (out of 4,267 ASIs). According to Figure 3.8, significant ASI Loss-only PPI networks composed 51.8\% of PIPE constructed networks followed by Gain-only, and mixed PPI networks composing 40.0\% and $8.4 \%$ respectively. From the 195 ASIs that have Gain-only PPI networks, 185 ASIs (95\%) sequences lose sequence regions (i.e. truncated) relative to their CIs. Similarly, out of all ASIs with mixed PPI networks (gained and lost IPs), 95\% have truncated sequences. However, for ASIs with Lose-only networks, the distribution of truncated and prolonged ASI sequences are $87 \%$ and $13 \%$ respectively. The results for gain-only and mixed PPI may point to the possibility that there may be a link between sequence region truncations and changes on PPI networks. Also, the average similarity score for all 494 ASIs from the results is around \%50 which is roughly $30 \%$ lower than the global average of the dataset. 


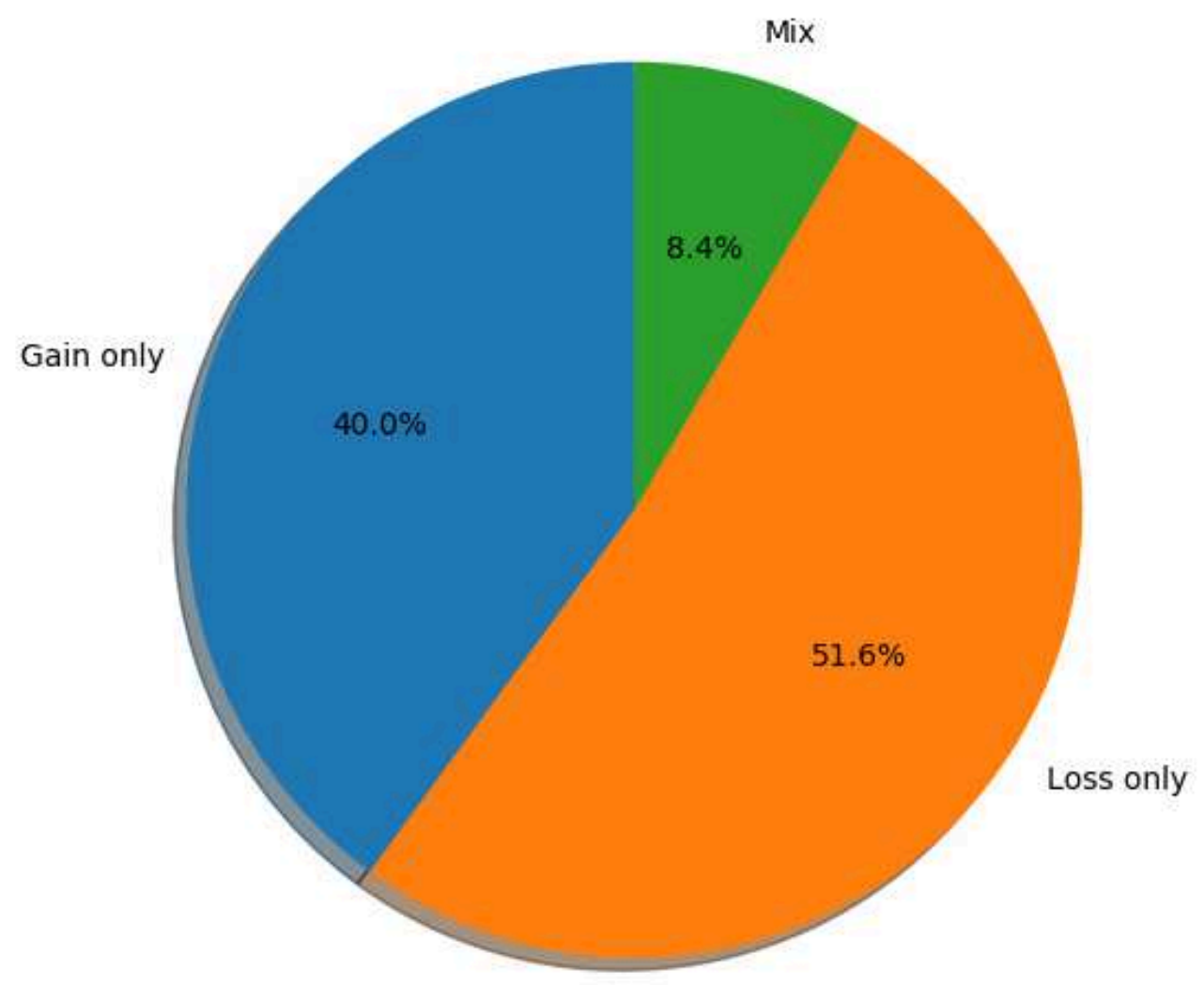

Figure 3.8: Breakdown of significant ASI PPI networks based on the gain or loss of interaction partners when compared to their CIs PPI networks

The results were then ranked according to the PPI network scoring equation. The ASIs from the top 10 significant PPI networks are summarized in Table 3.3. The outline of the PPI network construction pipeline analysis step is illustrated in Figure 3.9 . 


\begin{tabular}{|l||l|l|l||l|}
\hline & Gene & CI & ASI & ASI AS PPI network score \\
\hline 1 & HNRNPA1 & P09651 & P09651-3 & 1102 \\
2 & SUMO3 & P55854 & P55854-2 & 740 \\
3 & LMO2 & P25791 & P25791-4 & 175 \\
4 & UBE2L3 & P68036 & P68036-3 & 157 \\
5 & ZNF451 & Q9Y4E5 & Q9Y4E5-4 & 116 \\
6 & PRDM2 & Q13029 & Q13029-4 & 110 \\
7 & THOC2 & Q8NI27 & Q8NI27-2 & 89 \\
8 & ENDOV & Q8N8Q3 & Q8N8Q3-4 & 86 \\
9 & SUMO3 & P09651 & P09651-2 & 73 \\
10 & RBM4 & Q9BWF3 & Q9BWF3-4 & 64 \\
\hline
\end{tabular}

Table 3.3: Top 10 ASIs based on AS PPI network scores 


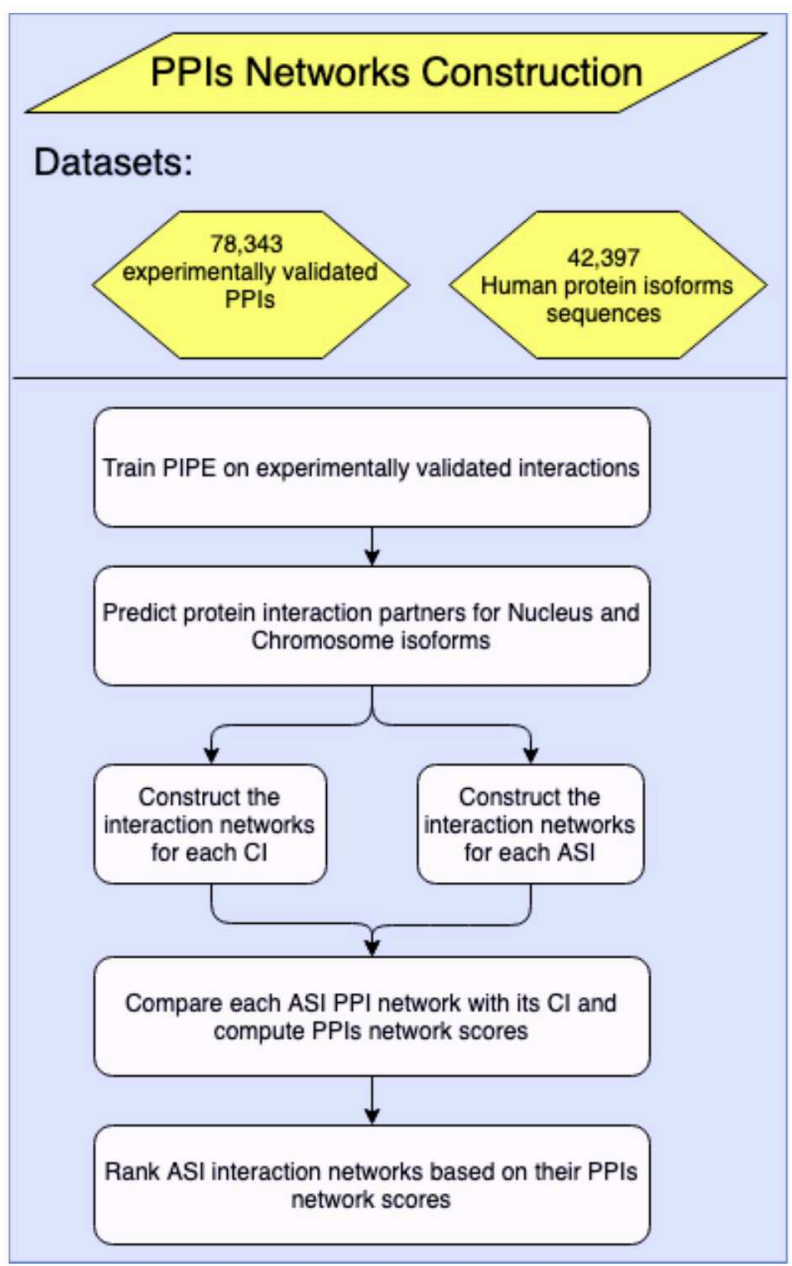

Figure 3.9: Protein-Protein Network construction pipeline step overview 


\subsection{Gene Ontology Term-Finder}

\subsubsection{Introduction}

Gene Ontology Terms or GO Terms, are descriptions of functions that occur within a living organism [49][8]. These GO terms are normally annotated to genes or gene products to help describe the functional aspects of the gene and its products [49][8]. GO terms are categorized into three categories. Namely, Biological process, Molecular Functional, and Cellular Components.

\subsubsection{Dataset}

For this project, the GO terms definitions set contained 46,661 GO terms, and was obtained from the GO Consortium [49][8]. The set includes 30,497 biological process Terms, 11,880 molecular function Terms, and 4,283 cellular component Terms (See Figure 3.10). On the other hand, the GO terms gene annotations were obtained from the Uniport Consortium totaling 261,517 annotations to 23,768 human genes [1].

\subsubsection{GO Term Finder}

Subsequent to getting the significant 494 ASI PPI networks, the resulting PPI networks are then functionally annotated by using gene ontology terms. For this experiment, we used the GO-Term Finder tool to find the common functional GO annotations among genes products that are participating in an interaction network [6]. 


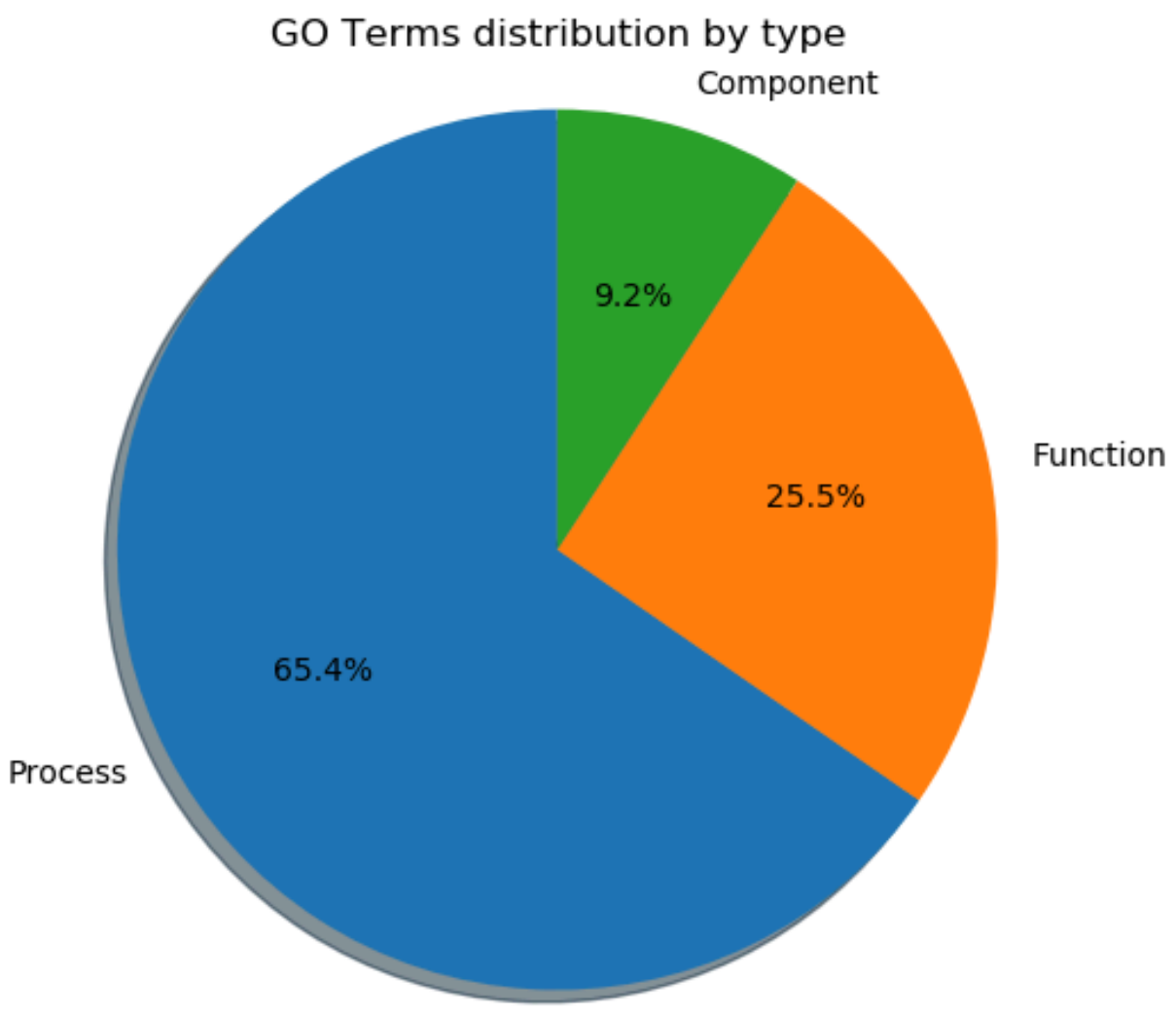

Figure 3.10: Gene Ontology Terms divided by category types

GO-Term Finder was introduced by Boyle et al. and it was originally designed to discover statistically significant shared GO annotations among a given set of yeast genes [6]. The tool takes two input files. The first is a text file that contains a list of genes to be investigated while the second is a file that contains yeast genes' GO annotations. Given these two input files the tool proceeds to find the common GO annotations among the query genes.

We extended the functionality of the tool to be capable of producing shared GO annotations for any organism including human genes and their protein isoforms. This was achieved by replacing the tool's file-parsing functions with scripts that allow for 
the integration of a multi-organism GO annotations' database as part of the tool's pipeline. The database is preloaded by python scripts that parse and fetch GO annotations from XML files downloaded from the Uniprot server. This modification allows any user of the tool to supply the organism symbol and the genes of interest to generate the shared GO annotation's reports. All the other tool's functionalities remained unchanged.

In this project, this tool is used as a way to compare the produced PPI networks based on GO annotations changes by identifying the annotations that are gained or lost by the ASI PPI network relative the CI's PPI network. This gives the ability to be able to distinguish between CIs and their ASIs PPI networks based on GO annotations and thus extending it to predicting functional differences between them.

Since alternatively spliced isoforms have no direct GO annotations, we resorted to inferring the gain/loss of function of a given ASI PPI network by analyzing the GO annotations of its interaction partners. The process followed by GO Term-Finder to produce the GO annotations for a network are listed as follows:

1. Given an ASI PPI network, GO-Term Finder will list the IPs in the PPI network and then fetch the GO annotations for each IP from the local database of annotations.

2. GO-Term Finder then proceeds to find the common GO annotations among all the interaction partners in the ASI interaction network.

3. Then the algorithm produces a list of annotations that are shared among all interaction partners in the ASI interaction network along with a p-value score for each annotation as a measure of confidence for the result. 
4. The same steps taken for the ASI interaction network (1 to 3 ) are repeated for its CI interaction network.

5. Having both lists of shared GO annotations for the CI and the ASI PPI networks produced, the final step is to compare the annotations in both lists and determine which annotations were gained, lost, or retained by the ASIs interaction network relative to the CIs interaction network.

Over all 494 significant ASI interaction networks found by PIPE, there were 20,323 predicted lost GO annotations relative to their CI PPI networks, and around 21,121 predicted gained annotations. In the upcoming section "Top Ten Literature Overlapped ASIs", we will review GO annotation findings in conjunction with PPI network results and tissue expression results in the light of studies from the literature. Figure 3.11 shows the pipeline GO analysis step summary.

\subsection{Tissue Expression Analysis}

\subsubsection{Introduction}

The human Protein Atlas is a project that started in 2003 just after the completion of the human genome project [51][50]. Whereas the human genome project objective was to map the human genome, the objective of the Human Atlas project is the mapping of the human proteome. The findings of this project are all open-source and freely accessible to researchers. The project focuses on three different areas in regard to mapping proteins, the first is the tissue Atlas where the focus is to discover the distribution of proteins across different tissues. Second is the cell Atlas whose 


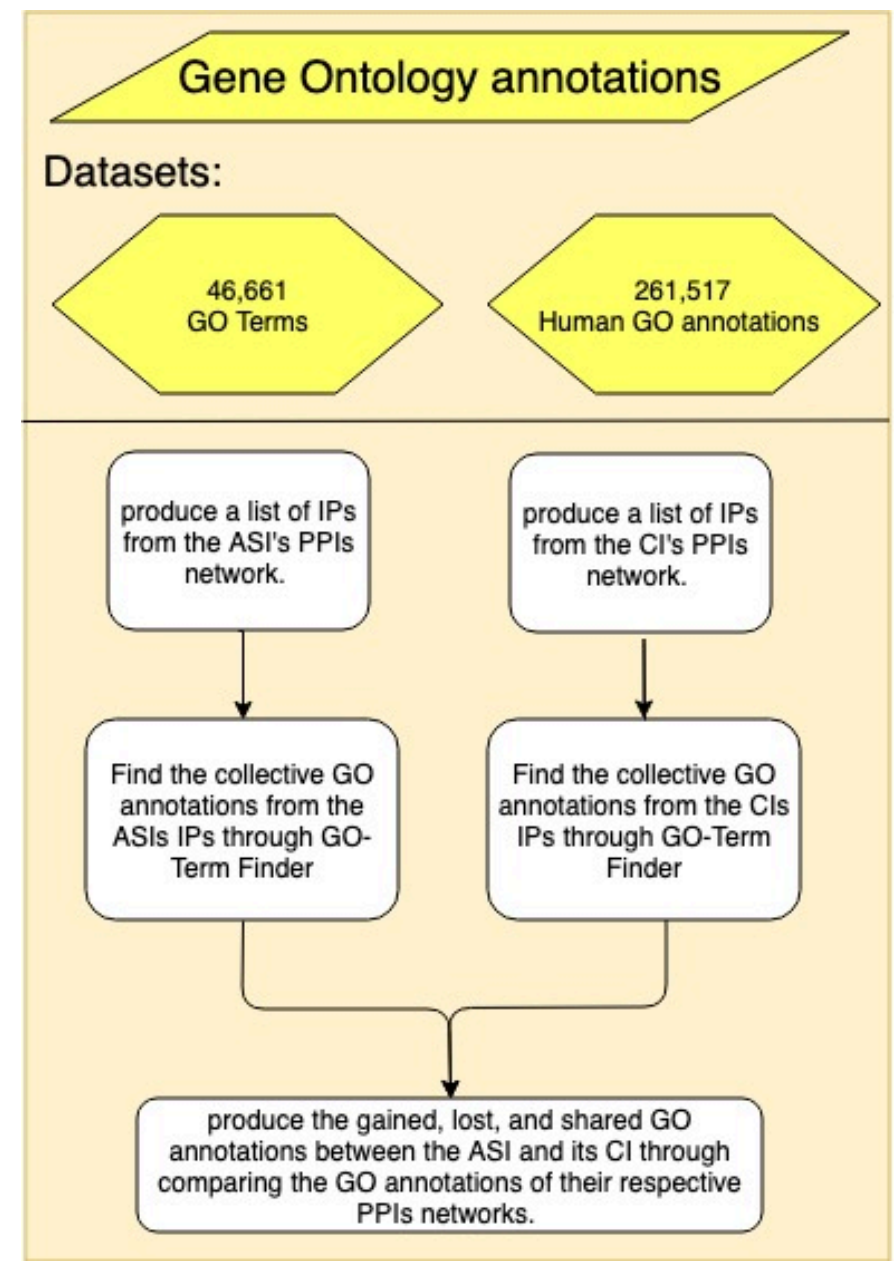

Figure 3.11: GO annotations pipeline step overview

objective is mapping proteins to subcellular locations. Finally the pathology Atlas tries to find links between proteins and cases of cancer [51][50]. For our experiment, we focus on the Tissue Atlas results and use the dataset for protein isoforms expression levels across different tissues. 


\subsubsection{Dataset}

The protein expression dataset contains RNA-seq protein isoforms expression levels from 37 different human tissue types [51][50]. The expression data comes from 22,357 different genes with a total of 160,093 protein isoforms. The number of samples (i.e. individuals) for each tissue type varies ranging from 13 samples for the colon tissue and 1 sample as such is the case for the epididymis tissue. For each transcript/protein isoform, the dataset outlines which gene it comes from and its expression value for each tissue type using the Transcript Per Million measurement (TPM).

\subsubsection{Dataset-cleanup \& Analysis steps}

The Analysis pipeline for the expression dataset follows the following steps:

1. The first step in analyzing the dataset was to remove tissue types that have less than three samples as the effect of measurement errors decreases as the sample count increases. This resulted in the removal of six tissue types and keeping 31 tissue types.

2. Next, the expression levels for each tissue type were averaged across its samples so that each transcript would only have one expression level for each tissue type.

3. Then each CI is compared to each of its ASIs and the difference between their expression levels is computed. If the difference between the two isoforms (CI and ASI) is below a defined threshold, the difference is considered insignificant. The difference between the expression levels is calculated by folds of change (i.e change of 2 folds). 
4. Subsequently, based on the difference of expression levels and a defined threshold, an ASI is marked as over expressed, under expressed or not changed in relation to the $\mathrm{CI}$ expression profile.

5. Finally, for each tissue type, a list of ASIs that have significant changes in relation to their CIs is produced.

\subsubsection{Results \& Discussion}

Since we are only considering alternatively spliced isoforms from nucleus and chromosome genes for this experiment, roughly only one quarter of the genes in the dataset were used. When conducting the comparison of the expression profile differences between the CIs and their ASIs in each of the 31 tissue types, only ASIs that have 10-fold expression increase or decrease when compared to their CI were considered significant. See Figure 3.12 for the significant ASI expression profile changes (overexpressed and under-expressed) for each tissue and Figure 3.13 for an overview of the tissue expression analysis pipeline step.

Globally, the average number of significant ASIs for all tissue types was 900 ASIs (out of 4,267 considered ASIs) with the Fallopian tube tissue type having the maximum of 1125 ASIs while the Salivary gland tissue type had the minimum of 440 ASIs. Roughly

only one fifth of significant ASIs for any tissue type were over-expressed compared to their relative CIs. This indicates that the process of alternative splicing possibly produces a much larger number of dormant/under-expressed or non-functional ASIs than functional ASIs.

The state of ASIs over-expression or under-expression, and ASIs sequence change types (addition, or truncation of sequence regions) do not seem to have a recognizable 

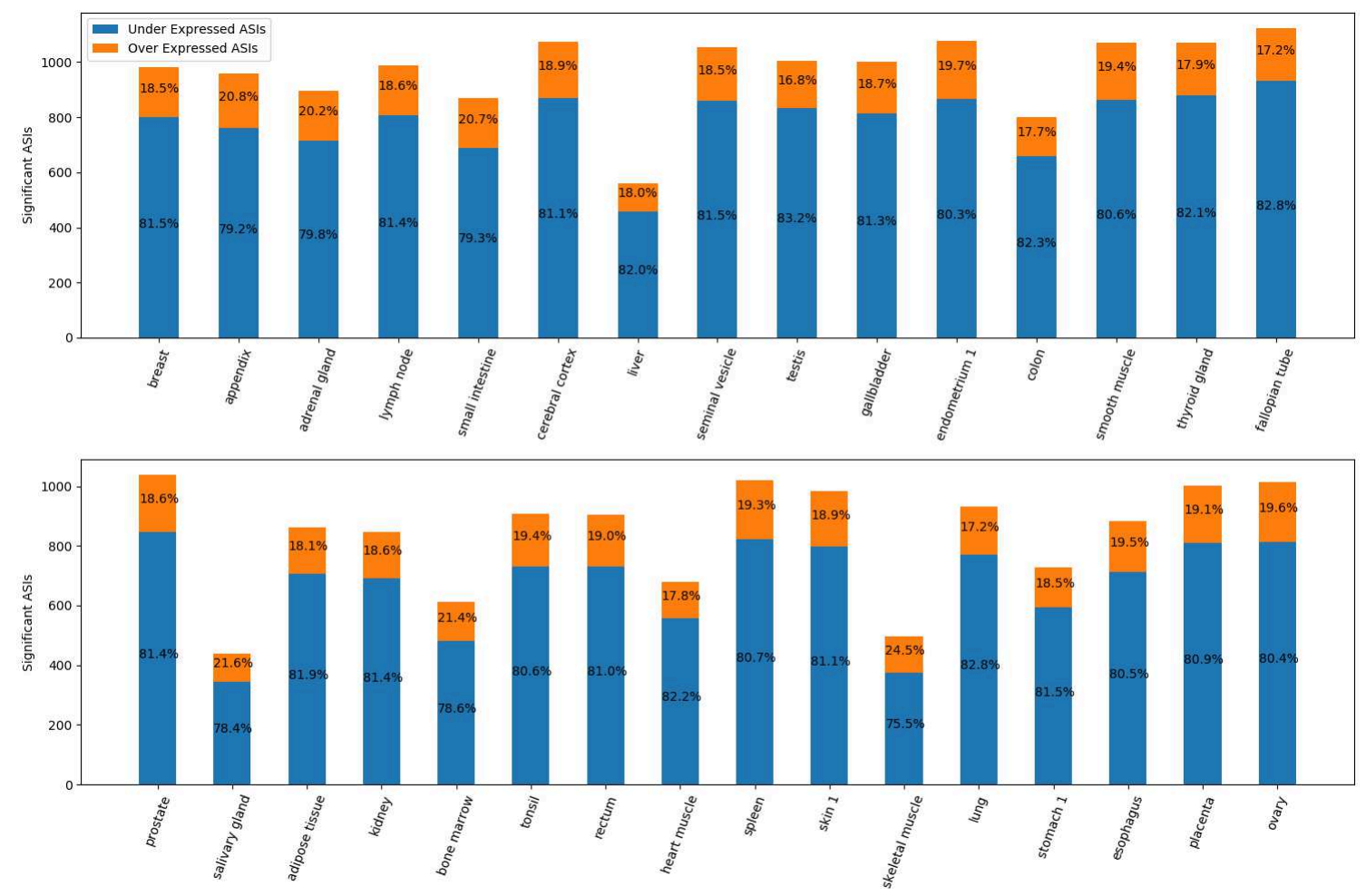

Figure 3.12: Frequencies of ASIs with significant overexpression/under-expression value changes for each of the 31 tissue types

link when it comes to tissue expression as ASIs with either additional or truncated sequence regions are distributed as expected in ASIs with significant tissue expression results (i.e $17 \%$ prolonged ASIs, $82 \%$ truncated ASIs). Similarly, significant ASIs across tissues had the expected $80 \%$ similarity score when compared to their CIs. 


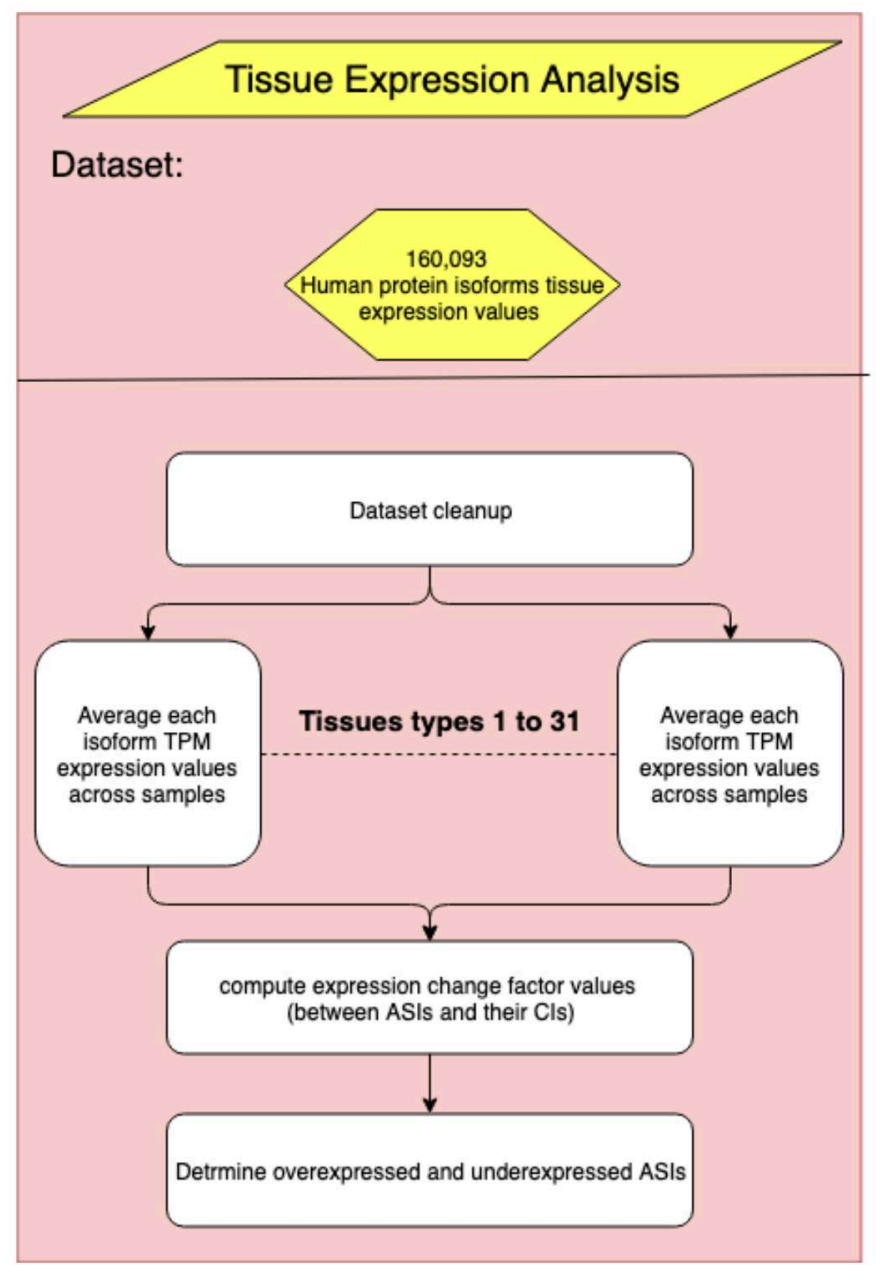

Figure 3.13: Overview of tissue expression analysis pipeline step

\subsection{PPI networks \& Tissue Expression Results Over- lap}

\subsubsection{Introduction}

The goal of overlapping ASIs with significant PPI network results and tissue expression results is to find ASIs that have significant PPI network differences relative to 
their CIs while also having a significant tissue expression fold change relative to the same CI. Once the analysis pipeline steps for the PPI network construction and the tissue expression analysis are both completed, ASIs from both sets are matched by their Uniprot IDs. Having overlaps between significant ASIs from both result sets allows for the deduction of conclusions about some of the possible links between PPI network changes and changes in tissue expression. This in turn would make possible the linking of the loss/gain of ASI functions relative to its CI.

\subsubsection{Example}

For example, assume you have Gene $e_{x}$ that has a canonical isoform $C I_{x}$ and one alternatively spliced isoform $A S I_{A} . C I_{x}$ has three interaction partners $I P_{1}, I P_{2}$ and $I P_{3}$ as part of its interaction network while $A S I_{A}$ has three different partners $I P_{4}, I P_{5}$, and $I P_{6}$. Assume that the expression levels for the heart tissue is $1 \mathrm{TPM}$ for $C I_{x}$ and is $40 \mathrm{TPM}$ for $A S I_{A}$. Having such a significant PPI change (ASI gained 3 IPs and lost 3 IPs) and a significant tissue expression profile difference (40 fold change) between $C I_{x}$ and $A S I_{A}$, we can draw a reasonable conclusion that $A S I_{A}$ and its interaction networks may have a functional role in the heart tissue that $C I_{x}$ and its interaction network may not have. This indicates that some functional differences may exist between the canonical isoform and one of its alternatively spliced isoforms.

\subsubsection{Overlap Types}

The PPI networks and the expression profiles of ASIs can be combined/overlapped in one of two methods/types: 
Method 1: ASIs with significant PPI network changes overlapped with ASIs with significant tissue expression fold changes.

In this method of overlap, each of the ASIs from the significant PPI networks is compared to the significant ASIs from the tissue expression analysis result set to see if the same ASI has a significant tissue expression fold change either by being significantly under-expressed or over-expressed (i.e. has a 10-fold change compared to the CI). Having a significant change in any of the 31 tissue types qualifies the ASI to be marked overlapped.

This type of overlap tries to answer the following question:

Given the 494 significant ASIs produced by the PPI network construction step, how many of the 494 ASIs have a significant tissue expression fold change when compared to their CI?

Method 2: ASIs with significant tissue expression fold changes from each tissue type overlapped with ASIs with significant PPI network changes.

In this type of overlap, each significant ASI from each tissue type is compared to the set of 494 significant ASIs produced by the network construction step.

This type of overlap tries to answer the following question:

Given n ASIs with significant expression fold changes of a tissue type $\mathrm{x}$, how many of $\mathrm{n}$ have a significant PPI network change when compared to their CI? 


\subsubsection{Statistical Significance Calculation Method}

After conducting the overlap and finding the significant hits that are shared among the two sets, a step of validating the results statistically takes place. Given the relationship between the PPI networks and the tissue expression levels, we are trying to answer the question of:

\section{How confident are we that the number of overlapped significant ASIs from both sets is not produced by chance?}

To answer this question, we use hypothesis testing, namely the goodness of fit t-test. For us, the null hypothesis $\left(H_{o}\right)$ and the alternative hypothesis $\left(H_{A}\right)$ are represented as follows:

$H_{o}$ : the number of overlapped significant ASIs from both sets is what is expected by chance

$H_{A}$ : The number of overlapped significant ASIs from both sets is not expected by chance

By defining the following symbols:

obs $s_{\text {yes }}=$ Observed ASI overlaps

$\exp _{\text {yes }}=$ Expected ASI overlaps

$o b s_{n o}=$ Observed ASI non-overlaps

$\exp _{n o}=$ Expected ASI non-overlaps

We can calculate the p-value through the following equation: 


$$
X^{2}=\frac{\left(o b s_{y e s}-e x p_{y e s}\right)^{2}}{e x p_{y e s}}+\frac{\left(o b s_{n o}-e x p_{n o}\right)^{2}}{e x p_{n o}}
$$

We reject the null hypothesis when we get $X^{2}$ score associated with a p-value that is less than 0.05. For this case, there is one degree of freedom and thus the corresponding chi score to a 0.05 p-value is 3.841 (i.e we reject when $X^{2} \geq 3.841$ ).

\subsubsection{Results \& Discussion}

\section{Method 1:}

Since we know that in the tissue expression analysis step that the number of considered ASIs is the same as that of the PPI network construction step which is 4,267 ASIs, and that the tissue expression analysis step result set produced 2,455 ASIs with at least one significant tissue expression factor change, we expect the overlap rate to be $57.5 \%$ by chance. 333 out of 494 ASIs with significant PPI networks have also a significant tissue expression factor change with at least one tissue type. 333 out of 494 is a statistically significant overlap count as demonstrated by the p-value produced by the following calculation:

$$
\begin{aligned}
& o b s_{\text {yes }}=333 \\
& \exp _{\text {yes }}=0.575 \times 494=284 \\
& o b s_{n o}=161 \\
& \exp _{\text {no }}=494 \exp _{\text {yes }}=210
\end{aligned}
$$

The calculation of $X^{2}$ : 


$$
\begin{aligned}
X^{2} & =\frac{(333-284)^{2}}{284}+\frac{(161-210)^{2}}{210} \\
& =8.45+11.43 \\
& =19.88
\end{aligned}
$$

By examining the chi-square distribution with one degree of freedom, we find that 19.88 correspondences to a p-value of $8.25 \times 10^{-5}$. Thus, we reject $H_{o}$ and accept $H_{A}$

Based on the results reported in Figure 3.8, the percentages of overlapped ASIs were $69 \%, 67 \%$, and $56 \%$ for Lose-only, Gain-only and mixed ASI PPI networks respectively. Out of the $69 \%$ overlapped ASIs with Lose-only PPI networks, 140 out of the 175 (80\%) were significantly under-expressed in at least one tissue type. On the other hand, for the $67 \%$ overlapped ASIs with Gain-only PPI networks, 20 out of $132(15 \%)$ were significantly over-expressed in at least one tissue type.

Method 2:

Determining the overlap distribution p-value for each tissue type result set is done in a similar fashion to that of method 1 . We know that the total number of significant ASIs with PPI network changes is 494 out of 4,267 or $11.5 \%$ out of all considered ASI PPI networks. Since the number of ASIs that have had their expression difference factors computed and been considered is the same as that of the PIPE dataset (i.e. $4,267)$, we can expect that the overlap rate with any tissue type's significant ASIs will be around $11.5 \%$ by chance.

Therefore, the calculation of the p-value for each tissue type result can be done using the same equation that was used in method 1. 
28 out of 31 tissue types had p-values values above the $99 \%$ confidence level. See Figure 3.14 which shows the level of statistical significance for each of the 31 tissue types along with the percentage of overlapped significant over-expressed/under-expressed ASIs.
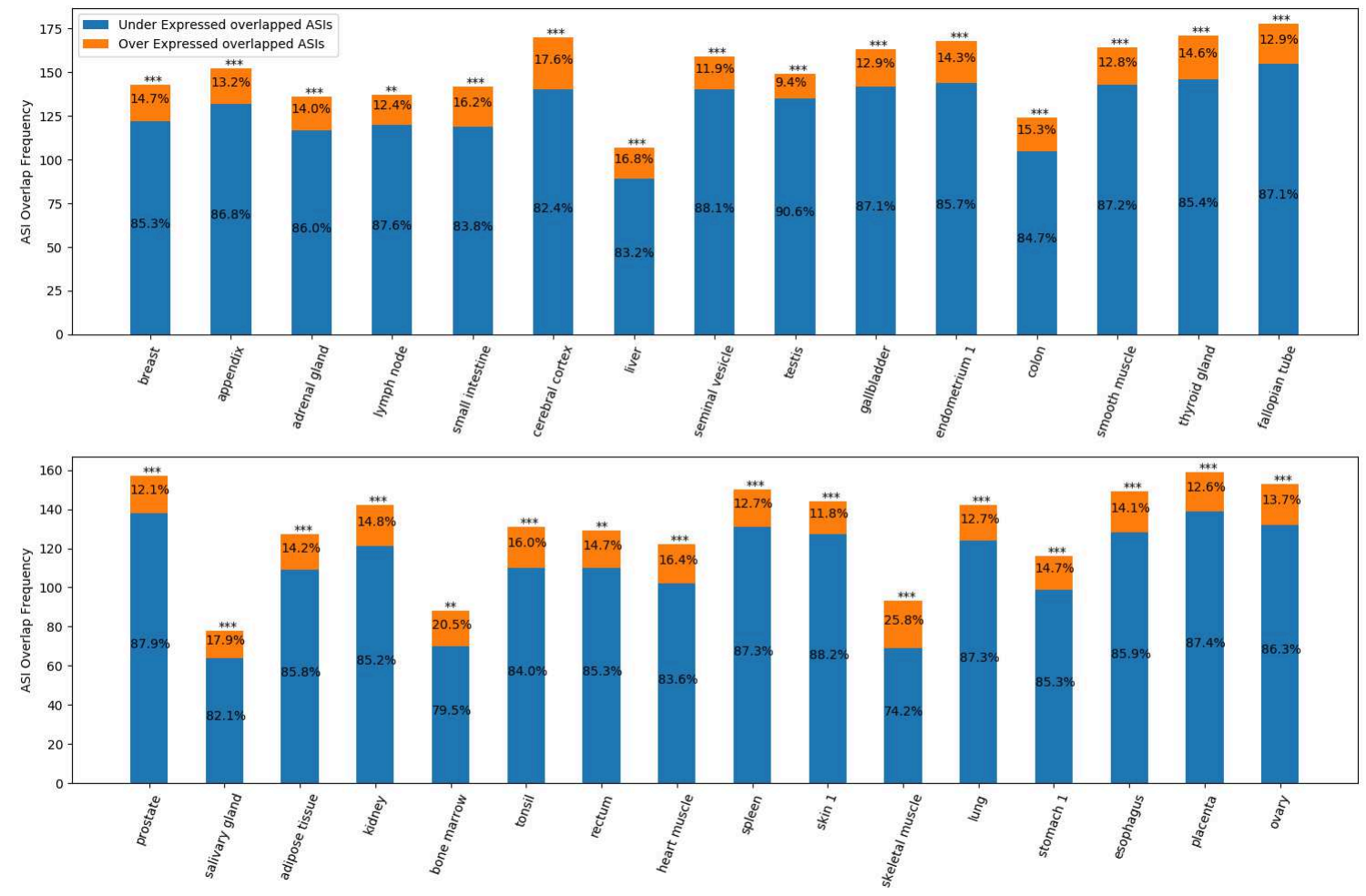

Figure 3.14: Frequencies of overlapped over-expressed/under-expressed ASIs for each of the 31 tissue types (90\%, 95\%, 99\% confidence levels are represented by ${ }^{*},{ }^{* * *}$, and ${ }^{* * *}$, respectively)

Generally, the number of significantly expressed ASIs in a given tissue type tends to correlate positively with the number of overlapped ASIs for the same tissue type (i.e high number of significant ASIs means high number of overlapped ASIs for a given tissue). By reviewing Figure 3.14, it is observed that the percentage of under- 
expressed ASIs that overlap is higher than that of ASIs that are over-expressed in almost all tissue types. This indicates that ASIs that have significant PPI network changes are more likely to be under-expressed rather than over-expressed.

\subsection{Pipeline \& Literature Results Overlap}

\subsubsection{Introduction}

In this section we will review the top 10 ASIs with significant PPI network changes in light of data from the literature. For each of the ASIs reported in Table 3.3 we will outline the overlap between the data reported from the literature and the data reported from the analysis pipeline results.

\subsubsection{Top Ten Literature Overlapped ASIs}

ASI\# 1: P09651-3 (HNRNPA1)

This gene has multiple molecular functions from modulating splice site selection in mRNA to packing mRNA and transporting it from the nucleus to the cytoplasm [38]. According to this study functions such as nucleotide binding, transferase activity and GTP binding remained preserved between the ASI and its CI.

ASI\# 2: P55854-2 (SUMO3)

SUM03 is a Ubiquitin-like protein that does not seem to be involved in protein 
degradation and is rather involved in cellular processes such as nuclear transport and signal transduction [48]. One of the functions were gained by the ASI (P55854-2) PPI network as opposed to its CI (P55854) is K channel regulator activity $(\mathrm{p}=0.0005)$. This affects the polarization of the cell membrane and subsequently causes contractions in different muscles. The ASI expression values were highly reduced in cardiac muscles (by 202-fold), smooth muscles (by 86fold), and skeletal muscles (by 72-fold) relative to the CI.

ASI\# 3: P25791-4 (LMO2)

LMO2 has various molecular functions and is involved in transcription regulation of genes [46]. Some molecular functions were preserved between the CI and the ASI including ribonucleotide binding, ATP binding, DNA-binding transcription activator activity and RNA polymerase II activating transcription factor binding.

Studies have shown in basal-type breast cancer specifically, high LMO2 expression was positively correlated with lymph node metastases in patients. According to the pipeline expression results, the ASI was under-expressed by 3-folds in the lymph node tissue compared to the CI [29].

ASI\# 4: P68036-3 (UBE2L3)

This protein acts with other ubiquitin-protein ligases and is involved in the selective degradation of proteins. However, functions such as ubiquitin-like protein transferase activity, ubiquitin protein ligase activity and ubiquitin-protein transferase activity were predicted to be lost in the ASI compared to the CI as per the pipeline results. 
According the pipeline expression results, the ASI was reported to be highly under-expressed in the cerebral cortex tissue (159-fold).

According to a study by Wang in 2012, UBE2L3 is associated with increased susceptibility to numerous autoimmune diseases when it is highly down regulated in cerebral cortex [32].

ASI\# 5: Q9Y4E5-4 (ZNF451)

ZNF451 is a gene that produces a small ubiquitin-like modifier-protein ligase and transferase which plays a role in modifying protein function by covalently attaching to and detaching from other proteins [7]. Functions such as protein tag, transcription cofactor binding and ubiquitin-like protein transferase activity are predicted have been lost by the ASI Q9Y4E5-4 of the gene according to the pipeline results.

A study by Hendriks et al. published in 2018 has shown SUMOylation in skeletal muscle and heart had the largest dependence on ZNF451 abundance [16]. However, brain, lung, spleen, and testis, only displayed a modest correlation. According to our expression data, the ASI showed the most reduction of expression values in heart muscle (15-fold) when compared to CIs expression data.

ASI\# 6: Q13029-4 (PRDM29)

Studies have shown a reduction of the expression level of the gene during the differentiation of myoblasts into myotubes in different muscles [?]. According to our study's expression data, the ASI has the highest over-expression value (compared to the CI) in skeleton muscle tissue which could induce functional changes. 
ASI\# 7: Q8NI27-2 (THOC2)

THOC2 is a gene that is required for efficient export of mature mRNA as well as for the process and the nuclear export of mRNA.

THOC2 also plays a role in the proper neuronal development. According to a study by Kumar et al. in 2015, variants in THOC2, which encode a subunit of TREX mRNA-export complex, cause syndromic intellectual disability (ID) and also cause reduced levels of THOC2 in skin fibroblasts in affected individuals [19]. According to the expression data from the pipeline analysis, the ASI is highly over-expressed with 16 -fold increase relative to the $\mathrm{CI}$ in the skin tissue.

ASI\# 8: Q8N8Q3-4 (ENDOV)

ENDOV is an Inosine-Specific Endoribonuclease that has strong preference to cleave single-stranded RNAs and tRNAs [53]. This gene was initially identified as an endodeoxyribonuclease involved in the DNA repair process [35]. Our study results show a shared function in ribonucleotide binding and purine ribonucleotide binding between the CI (Q8N8Q3) and its ASI (Q8N8Q3-4) and also a gained function in organic cyclic compound binding which is inline with the expected function of the gene.

ASI\# 9: P09651-2 (HNRNPA1)

As mentioned for the 2nd ASI, this gene contributes to mRNA splice site selection, packaging and transport from nucleus to cytoplasm. The data in the current study indicates that the corresponding ASI has gained a new function in macromolecular biosynthesis process and protein transport $\left(\mathrm{p}=4 \times 10^{-6}\right.$ and $\mathrm{p}=5 \times 10^{-6}$ respectively). In accord with this observation, the expression of 
the corresponding ASI is the highest in tissues including liver and spleen. One study further connects the activity of this gene to stress response by re-localizing stress granules in the cell [15]. Another study reports that the protein product of this gene contains a nuclear transport signal that works in a temperature (a type of stress) dependent manner [34].

ASI\# 10: Q9BWF3-4 (RBM4)

Is an RNA-binding factor involved in multiple aspects of cellular processes. For example, activates exon skipping of the PTB pre-mRNA during muscle cell differentiation and modulates muscle cell-specific exon selection of alpha tropomyosin [28]. The ASI was highly over-expressed relative to the CI in smooth muscle (47-fold) and cardiac muscle (12-fold) which is in accordance to its predicted gained function of actin binding and troponin $\mathrm{T}$ binding. 


\section{Chapter 4}

\section{Challenges, Conclusion, and Future Work}

\subsection{Challenges}

During the process of developing and running the analysis pipeline, numerous challenges were faced ranging from small hurdles to more major blockers that required a redesign of some elements of the pipeline. In the this section we review some of the issues and what was done to overcome them.

One of the major problems encountered while overlapping ASIs from the "Results Overlap" step, was to ensure that UniProt isoform IDs were correctly linked to the matching isoform IDs from the tissue expression dataset. Although both datasets contain data about the same human isoforms, each dataset uses its own ID schemes. To solve this issue, a third dataset that contains ID mappings of the two ID schemes had to be fetched from a UniProt server. Using the mapping from the third dataset, 
we were able to convert the majority of isoforms between the two sets; however, there were a few cases where a group of isoform IDs from the tissue dataset mapped to a single Uniprot ID mapping (many-to-one). In these cases, the results these isoform IDs are linked to would be manually validated or dropped from further analysis if necessary.

Another issue was the presence of a single isoform that is labeled as canonical for multiple genes. In this case, the gene and its related data would be dropped from the analysis as this is normally a consequence of a labeling error and could produce inaccurate results.

Lastly, running the "PPI ASI Network Construction" and the "Tissue Expression Analysis" steps of the pipeline were the bottle necks of the system. Initially, these two steps would take up to an hour (depending on the input parameters) to run and produce results. To speed-up the processing time, we modified the scripts such that the two slow steps would be run once with the most permissive parameter options and subsequently storing the output in an SQLite database. This would produce output results that would cover all possible parameter choices thus eliminating the need to run these steps again for subsequent analysis pipeline runs. As a result of this change, running the pipeline takes around a second.

\subsection{Conclusion}

In this thesis we investigate the effects of alternative splicing by analyzing the human AS genes localized in the nucleus and chromosome in terms of physical, functional, and tissue expression differences. Before starting the analysis pipeline, the human protein isoform sequences dataset was analyzed. It was found that AS occurs extensively in 
the human genome with roughly half the human genome undergoing AS. Also, the tendency of ASIs was found to be similar to their CIs in sequence structure; however, when they do differ, ASIs sequences were much more likely to be truncated when compared to their CIs sequences.

Subsequently, an analysis pipeline was constructed and used in analyzing the effects of alternative splicing on the nucleus and chromosome proteome. The pipeline had four major steps starting with Protein-Protein interaction networks construction for each ASI, then functionally annotating the results using the GO-Term Finder tool. Afterwards, ASI tissue expression factor changes were computed and were used in the final step of overlapping them with significant ASIs from the constructed PPI networks either by overlaying ASIs with PPI network changes results over each tissue.

Out of the considered ASIs in this study, almost $57.5 \%$ were found to have significant tissue expression factor changes while only $11.5 \%$ had significant PPI network changes when compared to their CIs. Nonetheless, there was no strong correlation between ASIs similarity scores, or sequence structure changes and patterns of expression. However, $80 \%$ of ASIs with significant lose-only PPI networks had truncated sequences and all 494 ASIs with significant PPI changes had an average of $50 \%$ sequence similarity score compared to the global similarity score of $79 \%$. These results can be investigated further to establish links between sequence structures and PPI networks behaviors. Overlapping results from the PPI networks with tissue expression results showed that ASIs with loss of interaction partners from PPI networks which are also under-expressed are more frequent in the results compared to ASIs that have PPI with gained IPs and are over-expressed.

Finally, the top ten ASIs with the highest AS PPI network scores were compared to the literature to confirm the findings of the pipeline. For all of the top 10 ASIs, 
different aspects of the data produced by the analysis pipeline overlapped with results found in the literature. LMO2, UBE2L3, and THOC2 are examples of genes that were reported to have links to diseases that manifest under certain conditions in relation to these genes. These conditions were met by each of the genes' ASI results that were produced by the analysis pipeline. On the other hand, for cases such as the HNRNPA1 (P09651-3 ASI) and ENDOV genes, the pipeline results for their ASIs were in line with their genes' expected functions, resulting in the ASI and the CI of each of the genes to have roughly the same predicted functions despite the changes in their PPI networks.

In conclusion, this study provided quantifiable measures that shed light on the degree to which AS products differ in terms of physical, functional, and tissue expression profiles. Also, three out of ten of the top cases reported by the pipeline results had literature evidence that indicates their involvement in diseases. These results are aimed at providing supporting data to researchers who studying AS in humans.

\subsection{Future Work}

Given that this was an exploratory study where the focus was more on the general effects of alternative splicing, most of the reported results in this study were general oversights of AS. A natural follow-up study will ideally be a more focused approach on a single aspect of AS such as a more deeper look into PPI network changes, tissue expression, or disease links. For example, identifying a gene or a couple of genes that are associated to a particular disease through AS could help understand AS in a more specific manner. Also, the current lack of a gold standard for alternatively 
spliced isoforms hinders the validation process of results. When large and reliable gold-standard datasets are available it would be ideal to use them to train a model that will be more accurate and would probably uncover more insights about AS. 


\section{Bibliography}

[1] Rolf Apweiler. The universal protein resource (UniProt) in 2010. Nucleic Acids Research, 38(SUPPL.1):D142-D148, jan 2009.

[2] Boris Babenko. Multiple instance learning: algorithms and applications. Technical Report, pages 1-19, 2008.

[3] Susan M Berget, Claire Moore, and Phillip A Sharp. Spliced segments at the 5' terminus of adenovirus 2 late mRNA* (adenovirus $2 \mathrm{mRNA}$ processing/5' tails on mRNAs/electron microscopy of mRNA-DNA hybrids). Biochemistry, 74(8):3171-3175, 1977.

[4] E. S. Bone, A. Tamm, and M. J. Hill. The production of urinary phenols by human gut bacteria. Journal of Medical Microbiology, 9(2), 1976.

[5] Jean-christophe Bourdon, Kenneth Fernandes, Fiona Murray-zmijewski, Geng Liu, Alexandra Diot, Dimitris P Xirodimas, Mark K Saville, and David P Lane. PDE10a - useful methods. 9(12):1-16, 2005.

[6] Elizabeth I. Boyle, Shuai Weng, Jeremy Gollub, Heng Jin, David Botstein, J. Michael Cherry, and Gavin Sherlock. GO::TermFinder - Open source software 
for accessing Gene Ontology information and finding significantly enriched Gene Ontology terms associated with a list of genes. Bioinformatics, 20(18):3710-3715, 2004.

[7] Laurent Cappadocia, Andrea Pichler, and Christopher D. Lima. Structural basis for catalytic activation by the human ZNF451 SUMO E3 ligase. Nature Structural and Molecular Biology, 22(12):968-975, 2015.

[8] S. Carbon, E. Douglass, N. Dunn, B. Good, N. L. Harris, S. E. Lewis, C. J. Mungall, S. Basu, R. L. Chisholm, R. J. Dodson, E. Hartline, P. Fey, P. D. Thomas, L. P. Albou, D. Ebert, M. J. Kesling, H. Mi, A. Muruganujan, X. Huang, S. Poudel, T. Mushayahama, J. C. Hu, S. A. LaBonte, D. A. Siegele, G. Antonazzo, H. Attrill, N. H. Brown, S. Fexova, P. Garapati, T. E.M. Jones, S. J. Marygold, G. H. Millburn, A. J. Rey, V. Trovisco, G. Dos Santos, D. B. Emmert, K. Falls, P. Zhou, J. L. Goodman, V. B. Strelets, J. Thurmond, M. Courtot, D. S. Osumi, H. Parkinson, P. Roncaglia, M. L. Acencio, M. Kuiper, A. Lreid, C. Logie, R. C. Lovering, R. P. Huntley, P. Denny, N. H. Campbell, B. Kramarz, V. Acquaah, S. H. Ahmad, H. Chen, J. H. Rawson, M. C. Chibucos, M. Giglio, S. Nadendla, R. Tauber, M. J. Duesbury, N. T. Del, B. H.M. Meldal, L. Perfetto, P. Porras, S. Orchard, A. Shrivastava, Z. Xie, H. Y. Chang, R. D. Finn, A. L. Mitchell, N. D. Rawlings, L. Richardson, A. Sangrador-Vegas, J. A. Blake, K. R. Christie, M. E. Dolan, H. J. Drabkin, D. P. Hill, L. Ni, D. Sitnikov, M. A. Harris, S. G. Oliver, K. Rutherford, V. Wood, J. Hayles, J. Bahler, A. Lock, E. R. Bolton, J. De Pons, M. Dwinell, G. T. Hayman, S. J.F. Laulederkind, M. Shimoyama, M. Tutaj, S. J. Wang, P. D’Eustachio, L. Matthews, J. P. Balhoff, S. A. Aleksander, G. Binkley, B. L. Dunn, J. M. Cherry, S. R. Engel, F. Gondwe, K. Karra, K. A. MacPherson, S. R. Miyasato, R. S. Nash, P. C. 
Ng, T. K. Sheppard, A. Shrivatsav Vp, M. Simison, M. S. Skrzypek, S. Weng, E. D. Wong, M. Feuermann, P. Gaudet, E. Bakker, T. Z. Berardini, L. Reiser, S. Subramaniam, E. Huala, C. Arighi, A. Auchincloss, K. Axelsen, G. P. Argoud, A. Bateman, B. Bely, M. C. Blatter, E. Boutet, L. Breuza, A. Bridge, R. Britto, H. Bye-A-Jee, C. Casals-Casas, E. Coudert, A. Estreicher, L. Famiglietti, P. Garmiri, G. Georghiou, A. Gos, N. Gruaz-Gumowski, E. Hatton-Ellis, U. Hinz, C. Hulo, A. Ignatchenko, F. Jungo, G. Keller, K. Laiho, P. Lemercier, D. Lieberherr, Y. Lussi, A. Mac-Dougall, M. Magrane, M. J. Martin, P. Masson, D. A. Natale, N. N. Hyka, I. Pedruzzi, K. Pichler, S. Poux, C. Rivoire, M. Rodriguez-Lopez, T. Sawford, E. Speretta, A. Shypitsyna, A. Stutz, S. Sundaram, M. Tognolli, N. Tyagi, K. Warner, R. Zaru, C. Wu, J. Chan, J. Cho, S. Gao, C. Grove, M. C. Harrison, K. Howe, R. Lee, J. Mendel, H. M. Muller, D. Raciti, K. Van Auken, M. Berriman, L. Stein, P. W. Sternberg, D. Howe, S. Toro, and M. Westerfield. The Gene Ontology Resource: 20 years and still GOing strong. Nucleic Acids Research, 47(D1):D330-D338, 2019.

[9] Louise T Chow, Richard E Gelinas, and Richard J Roberts. An Amazing Sequence Arrangement of Adenovirus RNA at the 5 'Ends. Cell, 12(September):18, 1977.

[10] FRANCIS CRICK. Central Dogma of Molecular Biology. Nature genetics, 227:561-563, 1970.

[11] Annachiara De Sandre-Giovannoli, Rafaëlle Bernard, Pierre Cau, Claire Navarro, Jeanne Amiel, Irène Boccaccio, Stanislas Lyonnet, Colin L. Stewart, Arnold Munnich, Martine Le Merrer, and Nicolas Lévy. Lamin A truncation in Hutchinson-Gilford progeria. Science, 300(5628):2055, 2003. 
[12] Mohamed Ali Ghadie, Luke Lambourne, Marc Vidal, and Yu Xia. Domain-based prediction of the human isoform interactome provides insights into the functional impact of alternative splicing. PLoS computational biology, 13(8):e1005717, 2017.

[13] Daniela C. Glatz, Dan Rujescu, Yesheng Tang, Frank J. Berendt, Annette M. Hartmann, Frank Faltraco, Carlyn Rosenberg, Christine Hulette, Kurt Jellinger, Harald Hampel, Peter Riederer, Hans J. Möller, Athena Andreadis, Kerstin Henkel, and Stefan Stamm. The alternative splicing of tau exon 10 and its regulatory proteins CLK2 and TRA2-BETA1 changes in sporadic Alzheimer's disease. Journal of Neurochemistry, 96(3):635-644, 2006.

[14] Mar Gonzàlez-Porta, Adam Frankish, Johan Rung, Jennifer Harrow, and Alvis Brazma. Transcriptome analysis of human tissues and cell lines reveals one dominant transcript per gene. Genome Biology, 14(7):R70, 2013.

[15] S. Guil, J. C. Long, and J. F. Caceres. hnRNP A1 Relocalization to the Stress Granules Reflects a Role in the Stress Response. Molecular and Cellular Biology, 26(15):5744-5758, 2006.

[16] Ivo A Hendriks, David Lyon, Michael L Nielsen, Dan Su, Niels H Skotte, Jeremy A Daniel, and Lars J Jensen. SUMOylation across species and organs. Nature Communications, (2018), 2018.

[17] N. H. Horowitz, David Bonner, H. K. Mitchell, E. L. Tatum, and G. W. Beadle. Genic Control of Biochemical Reactions in Neurospora. The American Naturalist, $79(783): 304-317,2002$. 
[18] National Human Genome Research Institute. Figure: Production of multiple protein isoforms from a single gene. https://upload.wikimedia.org/wikipedia/ commons/0/0a/DNA_alternative_splicing.gif. Accessed: 2019-04-19.

[19] Raman Kumar, Mark A Corbett, Bregje W M Van Bon, Joshua A Woenig, Lloyd Weir, Evelyn Douglas, Kathryn L Friend, Alison Gardner, Marie Shaw, Lachlan A Jolly, Chuan Tan, Matthew F Hunter, Anna Hackett, Michael Field, Elizabeth E Palmer, Melanie Leffler, Carolyn Rogers, Jackie Boyle, Melanie Bienek, Corinna Jensen, Griet Van Buggenhout, Hilde Van Esch, Katrin Hoffmann, Martine Raynaud, Huiying Zhao, Robin Reed, and Hao Hu. THOC2 Mutations Implicate mRNA-Export Pathway in X-Linked Intellectual Disability. The American Journal of Human Genetics, 97(2):302-310, 2015.

[20] Stuart E. Leff, Michael G. Rosenfeld, and Ronald M. Evans. Complex Transcriptional Units: Diversity in Gene Expression By Altrenative RNA Processing. Annual Reviewes, (55):1091-1117, 1986.

[21] Hong Dong Li, Rajasree Menon, Gilbert S. Omenn, and Yuanfang Guan. Revisiting the identification of canonical splice isoforms through integration of functional genomics and proteomics evidence. Proteomics, 14(23-24), 2014.

[22] Hong Dong Li, Rajasree Menon, Gilbert S. Omenn, and Yuanfang Guan. The emerging era of genomic data integration for analyzing splice isoform function. Trends in Genetics, 30(8):340-347, 2014.

[23] Hong-Dong Li, Gilbert S. Omenn, and Yuanfang Guan. A proteogenomic approach to understand splice isoform functions through sequence and expression-based computational modeling. Briefings in Bioinformatics, (August 2015):bbv109, 2016. 
[24] Huan Li, Ziyue Wang, Tianyi Ma, Gang Wei, and Ting Ni. Alternative splicing in aging and age-related diseases. Translational Medicine of Aging, 1:32-40, 2017.

[25] Wenyuan Li, Shuli Kang, Chun Chi Liu, Shihua Zhang, Yi Shi, Yan Liu, and Xianghong Jasmine Zhou. High-resolution functional annotation of human transcriptome: Predicting isoform functions by a novel multiple instance-based label propagation method. Nucleic Acids Research, 42(6):1-15, 2014.

[26] Wenyuan Li, Chun Chi Liu, Shuli Kang, Jian Rong Li, Yu Ting Tseng, and Xianghong Jasmine Zhou. Pushing the annotation of cellular activities to a higher resolution: Predicting functions at the isoform level. Methods, 93:110$118,2016$.

[27] Chien Liang Glenn Lin, Lynn A. Bristol, Lin Jin, Margaret Dykes-Hoberg, Thomas Crawford, Lora Clawson, and Jeffrey D. Rothstein. Aberrant RNA processing in a neurodegenerative disease: The cause for absent EAAT2, a glutamate transporter, in amyotrophic lateral sclerosis. Neuron, 20(3):589-602, 1998.

[28] J.-C. Lin and W.-Y. Tarn. Exon Selection in -Tropomyosin mRNA Is Regulated by the Antagonistic Action of RBM4 and PTB. Molecular and Cellular Biology, 25(22):10111-10121, 2005.

[29] Ye Liu, Zhaoyang Wang, Di Huang, Chao Wu, Huihui Li, Xin Zhang, Bin Meng, Zongjin Li, Tianhui Zhu, Shuang Yang, and Wei Sun. LMO2 promotes tumor cell invasion and metastasis in basal-type breast cancer by altering actin cytoskeleton remodeling. Oncotarget, 8(6):9513-9524, 2017. 
[30] Ziyu Liu, Yahong Wang, Shuling Wang, Jing Zhang, Fei Zhang, and Yun Niu. Nek2C functions as a tumor promoter in human breast tumorigenesis. International Journal of Molecular Medicine, 30(4):775-782, 2012.

[31] Tingjin Luo, Weizhong Zhang, Shang Qiu, Yang Yang, Dongyun Yi, Guangtao Wang, Jieping Ye, and Jie Wang. Functional Annotation of Human Protein Coding Isoforms via Non-convex Multi-Instance Learning. pages 345-354, 2017.

[32] Xingjie Ma, Junjie Zhao, Fan Yang, Haitao Liu, and Weibo Qi. Ubiquitin conjugating enzyme E2 L3 promoted tumor growth of NSCLC through accelerating p27kip1 ubiquitination and degradation. 8(48):84193-84203, 2017.

[33] Agath man. Figure: Traditional classification of basic types of alternative rna splicing events. https://upload.wikimedia.org/wikipedia/commons/a/ab/ Alt_splicing_bestiary2.jpg. Accessed: 2019-04-19.

[34] W. Matthew Michael, Mieyoung Choi, and Gideon Dreyfuss. A nuclear export signal in hnRNP A1: A signal-mediated, temperature-dependent nuclear protein export pathway. Cell, 83(3):415-422, 1995.

[35] Rongjuan Mi, Maria Alford-Zappala, Yoke W. Kow, Richard P. Cunningham, and Weiguo Cao. Human endonuclease V as a repair enzyme for DNA deamination. Mutation Research - Fundamental and Molecular Mechanisms of Mutagenesis, 735(1-2):12-18, 2012.

[36] Gilbert S. Omenn, Hong-Dong Li, Bharat Panwar, Rajasree Menon, Ridvan Eksi, and Yuanfang Guan. Genome-Wide Functional Annotation of Human ProteinCoding Splice Variants Using Multiple Instance Learning. Journal of Proteome Research, 15(6):1747-1753, 2016. 
[37] Eddie Park, Zhicheng Pan, Zijun Zhang, Lan Lin, and Yi Xing. The Expanding Landscape of Alternative Splicing Variation in Human Populations. American Journal of Human Genetics, 102(1):11-26, 2018.

[38] Maria Paola Paronetto, Tilman Achsel, Autumn Massiello, Charles E. Chalfant, and Claudio Sette. The RNA-binding protein Sam68 modulates the alternative splicing of Bcl-x. Journal of Cell Biology, 176(7):929-939, 2007.

[39] Catalin Patulea. Targeted Optimization of Computational and Classification Performance of a Protein-Protein Interaction Predictor. Number September. 2011.

[40] Sylvain Pitre, Frank Dehne, Albert Chan, Jim Cheetham, Alex Duong, Andrew Emili, Marinella Gebbia, Jack Greenblatt, Mathew Jessulat, Nevan Krogan, Xuemei Luo, and Ashkan Golshani. PIPE: A protein-protein interaction prediction engine based on the re-occurring short polypeptide sequences between known interacting protein pairs. BMC Bioinformatics, 7:1-15, 2006.

[41] Jose Manuel Rodriguez, Paolo Maietta, Iakes Ezkurdia, Alessandro Pietrelli, Jan Jaap Wesselink, Gonzalo Lopez, Alfonso Valencia, and Michael L. Tress. APPRIS: Annotation of principal and alternative splice isoforms. Nucleic Acids Research, 41(D1):110-117, 2013.

[42] Ambrish Roy, Alper Kucukural, and Yang Zhang. I-TASSER: A unified platform for automated protein structure and function prediction. Nature Protocols, $5(4): 725-738,2010$.

[43] Michael Sammeth, Sylvain Foissac, and Roderic Guigó. A general definition and nomenclature for alternative splicing events. PLoS Computational Biology, 4(8), 2008. 
[44] K. Scotlandi, M. Zuntini, M. C. Manara, M. Sciandra, A. Rocchi, S. Benini, G. Nicoletti, G. Bernard, P. Nanni, P. L. Lollini, A. Bernard, and P. Picci. CD99 isoforms dictate opposite functions in tumour malignancy and metastases by activating or repressing c-Src kinase activity. Oncogene, 26(46):6604-6618, 2007.

[45] James A. Shapiro. Revisiting the central dogma in the 21st century. Annals of the New York Academy of Sciences, 1178:6-28, 2009.

[46] Marie Claude Sincennes, Magali Humbert, Benoît Grondin, Véronique Lisi, Diogo F.T. Veiga, André Haman, Christophe Cazaux, Nazar Mashtalir, E. L.Bachir Affar, Alain Verreault, and Trang Hoang. The LMO2 oncogene regulates DNA replication in hematopoietic cells. Proceedings of the National Academy of Sciences of the United States of America, 113(5):1393-1398, 2016.

[47] Claudia Tammaro, Michela Raponi, David I. Wilson, and Diana Baralle. BRCA1 exon 11 alternative splicing, multiple functions and the association with cancer. Biochemical Society Transactions, 40(4):768-772, 2012.

[48] Michael H. Tatham, Ellis Jaffray, Owen A. Vaughan, Joana M.P. Desterro, Catherine H. Botting, James H. Naismith, and Ronald T. Hay. Polymeric Chains of SUMO-2 and SUMO-3 are Conjugated to Protein Substrates by SAE1/SAE2 and Ubc9. Journal of Biological Chemistry, 276(38):35368-35374, 2001.

[49] The Gene Ontology Consortium, Michael Ashburner, Catherine A Ball, Judith A Blake, David Botstein, Heather Butler, J Michael Cherry, Allan P Davis, Kara Dolinski, Selina S Dwight, Janan T Eppig, Midori A Harris, David P Hill, Laurie Issel-tarver, Andrew Kasarskis, Suzanna Lewis, John C Matese, Joel E Richardson, Gerald M Rubin, and Gavin Sherlock. Gene Ontology : tool for the unification of biology. Nature genetics, 25(1):25-29, 2011. 
[50] The Human Protein Atlas. https://www.proteinatlas.org/. Accessed: 201904-15.

[51] Mathias Uhlén, Linn Fagerberg, Bjö M. Hallström, Cecilia Lindskog, Per Oksvold, Adil Mardinoglu, Åsa Sivertsson, Caroline Kampf, Evelina Sjöstedt, Anna Asplund, Ing Marie Olsson, Karolina Edlund, Emma Lundberg, Sanjay Navani, Cristina Al Khalili Szigyarto, Jacob Odeberg, Dijana Djureinovic, Jenny Ottosson Takanen, Sophia Hober, Tove Alm, Per Henrik Edqvist, Holger Berling, Hanna Tegel, Jan Mulder, Johan Rockberg, Peter Nilsson, Jochen M. Schwenk, Marica Hamsten, Kalle Von Feilitzen, Mattias Forsberg, Lukas Persson, Fredric Johansson, Martin Zwahlen, Gunnar Von Heijne, Jens Nielsen, and Fredrik Pontén. Tissue-based map of the human proteome. Science, 347(6220), 2015.

[52] Frédérique Végran, Romain Boidot, Claire Oudin, Jean Marc Riedinger, Franck Bonnetain, and Sarab Lizard-Nacol. Overexpression of caspase-3s splice variant in locally advanced breast carcinoma is associated with poor response to neoadjuvant chemotherapy. Clinical Cancer Research, 12(19):5794-5800, 2006.

[53] Erik Sebastian Vik, Meh Sameen Nawaz, Pernille Strøm Andersen, Cathrine Fladeby, Magnar Bjørås, Bjørn Dalhus, and Ingrun Alseth. Endonuclease v cleaves at inosines in RNA. Nature Communications, 4, 2013.

[54] Hui Y. Xiong, Babak Alipanahi, Leo J. Lee, Hannes Bretschneider, Daniele Merico, Ryan K.C. Yuen, Yimin Hua, Serge Gueroussov, Hamed S. Najafabadi, Timothy R. Hughes, Quaid Morris, Yoseph Barash, Adrian R. Krainer, Nebojsa 
Jojic, Stephen W. Scherer, Benjamin J. Blencowe, and Brendan J. Frey. The human splicing code reveals new insights into the genetic determinants of disease. Science, 347(6218), 2015.

[55] In Seok Yang, Hyeonju Son, Sora Kim, and Sangwoo Kim. ISOexpresso: A web-based platform for isoform-level expression analysis in human cancer. $B M C$ Genomics, 17(1):1-14, 2016.

[56] Xinping Yang, Jasmin Coulombe-Huntington, Shuli Kang, Gloria M. Sheynkman, Tong Hao, Aaron Richardson, Song Sun, Fan Yang, Yun A. Shen, Ryan R. Murray, Kerstin Spirohn, Bridget E. Begg, Miquel Duran-Frigola, Andrew MacWilliams, Samuel J. Pevzner, Quan Zhong, Shelly A. Trigg, Stanley Tam, Lila Ghamsari, Nidhi Sahni, Song Yi, Maria D. Rodriguez, Dawit Balcha, Guihong Tan, Michael Costanzo, Brenda Andrews, Charles Boone, Xianghong J. Zhou, Kourosh Salehi-Ashtiani, Benoit Charloteaux, Alyce A. Chen, Michael A. Calderwood, Patrick Aloy, Frederick P. Roth, David E. Hill, Lilia M. Iakoucheva, Yu Xia, and Marc Vidal. Widespread Expansion of Protein Interaction Capabilities by Alternative Splicing. Cell, 164(4):805-817, 2016.

[57] Nazar Zaki, Sanja Lazarova-Molnar, Wassim El-Hajj, and Piers Campbell. Protein-protein interaction based on pairwise similarity. BMC Bioinformatics, 10:1-12, 2009. 